\title{
The converging-input converging-state property for Lur'e systems
}

\author{
Adam Bill $^{1}$ - Chris Guiver ${ }^{1}$ (D) . \\ Hartmut Logemann ${ }^{1}$. Stuart Townley ${ }^{2}$
}

Received: 24 August 2015 / Accepted: 19 December 2016 / Published online: 20 January 2017

(C) The Author(s) 2017. This article is published with open access at Springerlink.com

\begin{abstract}
Using methods from classical absolute stability theory, combined with recent results on input-to-state stability (ISS) of Lur'e systems, we derive necessary and sufficient conditions for a class of Lur'e systems to have the converging-input converging-state (CICS) property. In particular, we provide sufficient conditions for CICS which are reminiscent of the complex Aizerman conjecture and the circle criterion and connections are also made with small gain ISS theorems. The penultimate section of the paper is devoted to non-negative Lur'e systems which arise naturally in, for example, ecological and biochemical applications: the main result in this context is a sufficient criterion for a so-called "quasi CICS" property for Lur'e systems which, when uncontrolled, admit two equilibria. The theory is illustrated with numerous examples.
\end{abstract}

This work was supported by the UK EPSRC_Engineering and Physical Sciences Research Council-(Grant EP/I019456/1).

$凶$ Chris Guiver

c.guiver@bath.ac.uk

Adam Bill

adamrbill@gmail.com

Hartmut Logemann

h.logemann@bath.ac.uk

Stuart Townley

s.b.townley@ex.ac.uk

1 Department of Mathematical Sciences, University of Bath, Claverton Down, Bath BA2 7AY, UK

2 Environment and Sustainability Institute, College of Engineering, Mathematics and Physical Sciences, University of Exeter, Penryn Campus, Penryn TR10 9FE, UK 
Keywords Absolute stability · Circle criterion - Converging-input converging-state property $\cdot$ Input-to-state stability $\cdot$ Lur'e systems $\cdot$ Non-negative systems

Mathematics Subject Classification 37N35 - 93C10 - 93C15 - 93C35 - 93C80 • 93D05 $\cdot$ 93D09 $\cdot$ 93D20 $\cdot$ 93D25

\section{Introduction}

We consider forced Lur'e systems in continuous-time of the form

$$
\dot{x}(t)=A x(t)+B f(C x(t))+v(t), \quad x(0)=x^{0} \in \mathbb{R}^{n}, \quad t \geq 0,
$$

where $A, B$ and $C$ are appropriately sized matrices, $f$ is a (nonlinear) function, $x$ denotes the state and $v$ is a control function (also interpreted as and named a disturbance, forcing term or input). Differential equations of the form (1.1) often arise as closed-loop systems obtained by the application of output feedback with nonlinear "characteristic" $f$ to the linear system specified by $(A, B, C)$, namely

$$
\dot{x}(t)=A x(t)+B u(t)+v(t), \quad y(t)=C x(t), \quad u(t)=f(y(t)), \quad t \geq 0,
$$

where $u$ and $y$ denote the input and output variables, respectively, see Fig. 1. Lur'e systems are a common and important class of nonlinear systems and are at the centre of the classical subject of absolute stability theory which includes the well-known real and complex Aizerman conjectures, circle and Popov criteria, see [13,14, 18,20,23, $24,45,47]$. An absolute stability criterion for (1.1) is a sufficient condition for stability, usually formulated in terms of frequency-domain properties of the linear system given by $(A, B, C)$ and sector or boundedness conditions for $f$, guaranteeing stability for all nonlinearities $f$ satisfying these conditions.

Traditionally, Lyapunov approaches to the stability theory of systems of the form (1.1) consider the uncontrolled $(v=0)$ case, whilst Lur'e systems with forcing (usually acting through $B$, that is, $v$ is of the form $v=B w$ for some $w$ ) have been studied using the input-output framework initiated by Sandberg and Zames in the 1960s; see, for example, $[11,45]$. More recently, forced Lur'e systems have been analyzed in the context of input-to-state stability (ISS) theory, see [4,19,20,36]. Whilst ISS is a concept for general controlled nonlinear systems (first formulated in [38]), in our context, ISS is a stability property of an equilibrium pair of (1.1), where $\left(x^{\mathrm{e}}, v^{\mathrm{e}}\right)$ is an equilibrium pair if $A x^{\mathrm{e}}+B f\left(C x^{\mathrm{e}}\right)+v^{\mathrm{e}}=0$. Loosely speaking, $\left(x^{\mathrm{e}}, v^{\mathrm{e}}\right)$ is ISS if the

Fig. 1 Block diagram of the controlled Lur'e system (1.1)

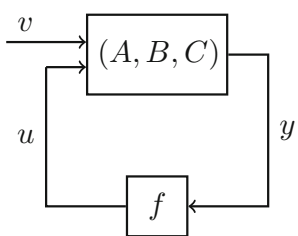


map $\left(x^{0}-x^{\mathrm{e}}, v-v^{\mathrm{e}}\right) \mapsto x\left(t ; x^{0}, v\right)-x^{\mathrm{e}}$ has "nice" boundedness and asymptotic properties (see (2.2)), where $x\left(\cdot ; x^{0}, v\right)$ denotes the solution of (1.1). In particular, if an equilibrium pair $\left(x^{\mathrm{e}}, v^{\mathrm{e}}\right)$ is ISS, then $x\left(t ; x^{0}, v\right) \rightarrow x^{\mathrm{e}}$ as $t \rightarrow \infty$ for all $x^{0} \in \mathbb{R}^{n}$ and all (essentially) bounded $v$ such that $\lim _{t \rightarrow \infty} v(t)=v^{\mathrm{e}}$. For surveys of ISS theory the reader is referred to $[9,42]$.

In the present paper, we investigate the following problem (and variations thereof): given $v^{\infty} \in \mathbb{R}^{n}$, find conditions (necessary or sufficient) for the existence of $x^{\infty} \in \mathbb{R}^{n}$ such that, for every $x^{0}$ and every $v$ with $v(t) \rightarrow v^{\infty}$ as $t \rightarrow \infty$, the solution $x$ of (1.1) converges to $x^{\infty}$. In particular, we consider the so-called converging-input converging-state (CICS) property: (1.1) is said to have the CICS property if, for every $v^{\infty} \in \mathbb{R}^{n}$, there exists $x^{\infty} \in \mathbb{R}^{n}$ such that $\lim _{t \rightarrow \infty} x(t)=x^{\infty}$ for all $x^{0}$ and all inputs $v$ converging to $v^{\infty}$.

For background and motivation, we comment that if (1.1) is linear and asymptotically stable, that is, for some matrix $F$, we have that $f(z)=F z$ and $A+B F C$ is Hurwitz (meaning every eigenvalue has negative real part), then (1.1) has the CICS property. Indeed, it is well known that, for given $v^{\infty}$, the state $x$ and output $y$ of (1.1) have respective limits

$$
x^{\infty}:=-(A+B F C)^{-1} v^{\infty} \text { and } y^{\infty}:=C x^{\infty}=-C(A+B F C)^{-1} v^{\infty}
$$

for every initial vector $x^{0}$ and every $v$ converging to $v^{\infty}$. The matrices $-(A+B F C)^{-1}$ and $-C(A+B F C)^{-1}$ are sometimes referred to as "steady-steady gains" and provide linear maps $v^{\infty} \mapsto x^{\infty}$ and $v^{\infty} \mapsto y^{\infty}$. If system (1.1) has the CICS property, then the steady-steady gain concept extends to Lur'e systems in the sense that there are explicit formulae for the nonlinear functions $v^{\infty} \mapsto x^{\infty}$ and $v^{\infty} \mapsto y^{\infty}$ which map input limits to state limits and output limits, respectively (see Sect. 4).

The main contribution of this paper is the establishment of sufficient conditions for the CICS property which are reminiscent of the complex Aizerman conjecture $[17,18,20,36]$, the circle criterion for ISS $[19,20,36]$ and the "nonlinear" ISS smallgain condition for Lur'e systems [36] and involve the transfer function matrix of the linear system $(A, B, C)$ and an incremental condition (in terms of norm or sector inequalities) on the nonlinearity $f$. Recent ISS results for Lur'e systems [36] play a key role in the development of the CICS theory in Sect. 4. We demonstrate that our sufficient CICS conditions also ensure that the nonlinear steady-state gains are continuous maps-further mirroring the linear case. We emphasize that CICS is a system property, whereas ISS relates to stability properties of an equilibrium pair of (1.1). In contrast to linear systems, Lur'e systems which admit a globally asymptotically stable equilibrium when uncontrolled $(v=0)$ need not have the CICS property. Indeed, there may exist convergent inputs such that, for some initial states, the corresponding state trajectory is asymptotically divergent (see part (b) of Example 4.5).

In certain circumstances, it is of interest to relax the CICS concept and restrict attention to convergent forcing terms $v$ with limits $v^{\infty}$ belonging to a subset of $\mathbb{R}^{n}$, perhaps just a singleton. For example, assuming that $f(0)=0$, the so-called 0-CICS property requires that $x\left(t ; x^{0}, v\right) \rightarrow 0$ as $t \rightarrow \infty$ for every $x^{0} \in \mathbb{R}^{n}$ and every $v \in L^{\infty}\left(\mathbb{R}_{+}, \mathbb{R}^{n}\right)$ such that $v(t) \rightarrow 0$ as $t \rightarrow \infty$. In deriving sufficient conditions for 
the "global" CICS property, we present a "local" CICS result in Theorem 4.3 (local in the limiting values $v^{\infty}$ of the forcing functions $v$ ), see also Examples 4.6.

In the context of general nonlinear systems, CICS-type properties (including 0CICS) have been studied in $[2,34,41]$. Concepts related to or reminiscent of the CICS property have been introduced in $[3,40]$. Whilst $[2,3,34,40]$ have little overlap with the material presented here, [41] plays an important role in the proof of statement (1) of Theorem 4.3, one of the main results in the current paper. To the best of our knowledge, there is not much previous work on CICS properties for Lur'e systems, exceptions include $[7,31,35]$. Of these works, [35] is, by some margin, the closest in spirit to the present paper and we provide detailed comments on the relation of the contribution in [35] to our results after the proof of Corollary 4.16. The papers [7,31] develop stability criteria from the perspective of incremental stability and convergent dynamics $[1,30,33,46]$ and touch upon aspects of CICS for Lur'e systems, but there is very little intersection with the systematic theory developed here.

We also study the CICS property for a class of Lur'e systems which is a variation of (1.1), namely

$$
\dot{x}(t)=A x(t)+B f(C x(t)-v(t)), \quad t \geq 0 \quad x(0)=x^{0} \in \mathbb{R}^{n},
$$

where the interpretation of the terms in (1.2) is the same as that in (1.1). System (1.2) can be thought of as a closed-loop system obtained by linear feedback applied to the linear system $(A, B, C)$ subjected to an input nonlinearity $f$ :

$$
\dot{x}(t)=A x(t)+B f(w(t)), \quad y(t)=C x(t), \quad w(t)=y(t)-v(t) \quad t \geq 0 .
$$

see Fig. 2. We derive a CICS criterion for Lur'e systems of the form (1.2) and use it to generalize a well-known result on integral control for linear systems to this class of nonlinear systems.

Furthermore, we consider a class of non-negative (also known as positive) Lur'e systems, cf. [6,36,37,44]. As an instance of a positive control system [15], these arise naturally in a variety of applied contexts: a common key feature is that their state variables, which may represent population abundances, chemical concentrations or economical quantities (such as prices) are, necessarily, non-negative. In a population model, the nonlinear term $f$ may describe Allee effects [8] or density-dependent recruitment owing to decreased survival rates or increased competition for resources at lower and higher population abundances, respectively. In a chemical reaction model, $f$ may describe a nonlinear reaction rate between certain reagents. Unforced biological, ecological and chemical models often admit (at least) two equilibria: the zero equilibrium and some non-zero equilibrium, the latter corresponding to the co-existence of populations or chemical compounds. The control $v$ in (1.1) may model immigration

Fig. 2 Block diagram of the controlled Lur'e system (1.2)

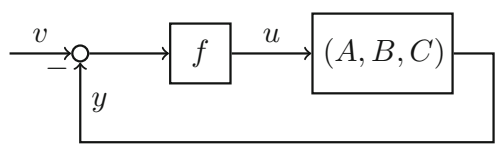


in a population model or the addition of a new reagent in a chemical reaction model. Our main result for non-negative Lur'e systems is a sufficient condition for a "quasi CICS" property which, for zero control $v=0$, have two equilibria (see Theorem 6.6). In this context, we shall make contact with recent work [6] on stability properties of non-negative Lur'e systems: a certain "repeller" or "persistence" property established in [6] will play a pivotal role in the proof of Theorem 6.6.

The paper is organized as follows. In Sects. 2 and 3, we discuss a number of preliminaries and prove necessary conditions for CICS, respectively. Section 4 is devoted to sufficient conditions for the CICS property for (1.1), the main result being Theorem 4.3, from which several CICS criteria are derived as corollaries. These criteria have the flavour of well-known absolute stability results (complex Aizerman conjecture, circle criterion and small gain). Sections 5 and 6 consider systems of the form (1.2) and non-negative versions of (1.1), respectively. We present some concluding comments in Sect. 7.

Notation and terminology For a set $S$, the symbol \#S denotes the cardinality of $S$ (if $S$ is infinite, then we write $\# S=\infty$ ). The set of positive integers is denoted by $\mathbb{N}$ and $\mathbb{R}$ and $\mathbb{C}$ denote the fields of real and complex numbers, respectively. We set $\mathbb{R}_{+}:=\{r \in \mathbb{R}: r \geq 0\}$. For $n \in \mathbb{N}, \mathbb{R}^{n}$ and $\mathbb{C}^{n}$ denote the usual real and complex $n$ dimensional vector spaces, respectively, both equipped with the 2-norm denoted by $\|\cdot\|$.

For $m \in \mathbb{N}$, let $\mathbb{R}^{n \times m}$ and $\mathbb{C}^{n \times m}$ denote the normed linear spaces of $n \times m$ matrices with real and complex entries, respectively, both equipped with the operator norm induced by the 2-norm, also denoted by $\|\cdot\|$. Given $M \in \mathbb{R}^{m \times n}$, we let im $M$ denote the image of $M$, that is, the linear subspace spanned by the columns of $M$. A matrix $M \in \mathbb{C}^{n \times n}$ is said to be Hurwitz if all its eigenvalues have negative real parts. Note that Hurwitz matrices are necessarily invertible. If $M$ is additionally real, with components $m_{i j}$, then it is said to be reducible if there exist non-empty disjoint subsets $J_{1}, J_{2} \subseteq$ $\{1, \ldots, n\}$ such that $J_{1} \cup J_{2}=\{1, \ldots, n\}$ and $m_{i j}=0$ for all $(i, j) \in J_{1} \times J_{2}$. We say that $M$ is irreducible if $M$ is not reducible. We refer the reader to [5,27] for more details on irreducible matrices.

Let $M=\left(m_{i j}\right) \in \mathbb{R}^{n \times m}$ and $N=\left(n_{i j}\right) \in \mathbb{R}^{n \times m}$. We write

$$
\begin{array}{ll}
M \geq N & \text { if } m_{i j} \geq n_{i j} \text { for all } i \text { and } j, \\
M>N & \text { if } M \geq N \text { and } M \neq N, \\
M \gg N & \text { if } m_{i j}>n_{i j} \text { for all } i \text { and } j .
\end{array}
$$

We say that $M$ is non-negative if $M \geq 0$. The matrix $M$ is called positive if $M \gg 0$. A square matrix $M=\left(m_{i j}\right) \in \mathbb{R}^{n \times n}$ is said to be Metzler (or essentially non-negative or quasi positive) if all its off-diagonal entries of $M$ are non-negative, that is, $m_{i j} \geq 0$ for all $1 \leq i, j \leq n$ with $i \neq j$. It is well known (and straightforward to prove) that $M \in \mathbb{R}^{n \times n}$ is Metzler if, and only if, $\mathrm{e}^{M t} \xi \in \mathbb{R}_{+}^{n}$ for all $\xi \in \mathbb{R}_{+}^{n}$ and all $t \geq 0$ (see, for example, [26]).

For $K \in \mathbb{C}^{m \times p}$ and $r>0$, set

$$
\mathbb{B}_{\mathbb{C}}(K, r):=\left\{Z \in \mathbb{C}^{m \times p}:\|Z-K\|<r\right\},
$$

the open (complex) ball in $\mathbb{C}^{m \times p}$, centred at $K$ and of radius $r$. 
A square rational matrix-valued function $s \mapsto \mathbf{H}(s)$ of a complex variable $s$ is said to be positive real if for every $s \in \mathbb{C}$ with $\operatorname{Re} s \geq 0$, which is not a pole of $\mathbf{H}$, the matrix $[\mathbf{H}(s)]^{*}+\mathbf{H}(s)$ is positive semi-definite. As usual, if $\mathbf{H}$ is a proper rational matrix-valued function which does not have any poles in the closed right-half plane $\operatorname{Re} s \geq 0$, then we define its $H^{\infty}$-norm by

$$
\|\mathbf{H}\|_{H^{\infty}}:=\sup _{\operatorname{Re} s \geq 0}\|\mathbf{H}(s)\|=\sup _{\omega \in \mathbb{R}}\|\mathbf{H}(i \omega)\| .
$$

More details on $H^{\infty}$-norms can be found in, for example, [29].

We recall the definitions of certain classes of comparison functions. Let $\mathcal{K}$ denote the set of all continuous functions $\varphi: \mathbb{R}_{+} \rightarrow \mathbb{R}_{+}$such that $\varphi(0)=0$ and $\varphi$ is strictly increasing. Moreover,

$$
\mathcal{K}_{\infty}:=\{\varphi \in \mathcal{K}: \varphi(s) \rightarrow \infty \text { as } s \rightarrow \infty\}
$$

We denote by $\mathcal{K} \mathcal{L}$ the set of functions $\psi: \mathbb{R}_{+} \times \mathbb{R}_{+} \rightarrow \mathbb{R}_{+}$with the following properties: $\psi(\cdot, t) \in \mathcal{K}$ for every $t \geq 0$, and $\psi(s, \cdot)$ is non-increasing with $\lim _{t \rightarrow \infty} \psi(s, t)=0$ for every $s \geq 0$. Following the convention of [39], we do not impose continuity in the definition of a $\mathcal{K} \mathcal{L}$ function. By [39, Proposition 7], it follows that a discontinuous $\mathcal{K} \mathcal{L}$-function can be bounded from above by a continuous one. For more details on comparison functions the reader is referred to [22].

The linear space of (equivalence classes of) locally essentially bounded functions $f: \mathbb{R}_{+} \rightarrow \mathbb{R}^{n}$ is denoted by $L_{\mathrm{loc}}^{\infty}\left(\mathbb{R}_{+}, \mathbb{R}^{n}\right)$. If $f \in L_{\mathrm{loc}}^{\infty}\left(\mathbb{R}_{+}, \mathbb{R}^{n}\right)$, then

$$
\|f\|_{L^{\infty}(0, t)}:=\operatorname{ess} \sup _{\tau \in[0, t]}\|f(\tau)\|<\infty \quad \forall t \geq 0 .
$$

As usual, $L^{\infty}\left(\mathbb{R}_{+}, \mathbb{R}^{n}\right)$ denotes the space of all essentially bounded functions $\mathbb{R}_{+} \rightarrow$ $\mathbb{R}^{n}$. For $f \in L^{\infty}\left(\mathbb{R}_{+}, \mathbb{R}^{n}\right)$, we write

$$
\|f\|_{L^{\infty}}:=\|f\|_{L^{\infty}(0, \infty)}=\underset{\tau \in[0, \infty)}{\operatorname{ess} \sup }\|f(\tau)\| .
$$

Finally, we use the symbol $\theta$ to denote the constant function $\mathbb{R}_{+} \rightarrow \mathbb{R}$ given by $\theta(t)=1$ for all $t \geq 0$.

\section{Preliminaries and definitions}

Consider the forced Lur'e system (1.1), where $A \in \mathbb{R}^{n \times n}, B \in \mathbb{R}^{n \times m}, C \in \mathbb{R}^{p \times n}, f$ : $\mathbb{R}^{p} \rightarrow \mathbb{R}^{m}$ is locally Lipschitz, $v \in L_{\mathrm{loc}}^{\infty}\left(\mathbb{R}_{+}, \mathbb{R}^{n}\right)$ is a control (forcing, input) function. If $v=0$, then we will refer to (1.1) as the uncontrolled system (1.1). Frequently, the input $v$ will be of the form $v=E w$, where $E \in \mathbb{R}^{n \times q}$ and $w \in L_{\mathrm{loc}}^{\infty}\left(\mathbb{R}_{+}, \mathbb{R}^{q}\right)$. If $q=m$ and $E=B$, then (1.1) can be written in the form

$$
\left.\begin{array}{l}
\dot{x}(t)=A x(t)+B u(t), \quad x(0)=x^{0} \in \mathbb{R}^{n}, \quad y(t)=C x(t) \\
u(t)=v(t)+f(y(t))
\end{array}\right\} \quad t \geq 0 .
$$


Let $x\left(\cdot ; x^{0}, v\right)$ denote the unique maximally defined forward solution of the initialvalue problem (1.1). We say that $\left(x^{\mathrm{e}}, v^{\mathrm{e}}\right) \in \mathbb{R}^{n} \times \mathbb{R}^{n}$ is an equilibrium pair of (1.1) if $A x^{\mathrm{e}}+B f\left(C x^{\mathrm{e}}\right)+v^{\mathrm{e}}=0$, that is, if $x^{\mathrm{e}}$ is an equilibrium of the (autonomous) differential equation

$$
\dot{x}(t)=A x(t)+B f(C x(t))+v^{\mathrm{e}}, \quad x(0)=x^{0} \quad t \geq 0 .
$$

It is clear that if, for some $v^{\infty} \in \mathbb{R}^{n}$ and $x^{0} \in \mathbb{R}^{n}, x\left(t ; x^{0}, v^{\infty} \theta\right)$ converges to $x^{\infty}$ as $t \rightarrow \infty$, then $\left(x^{\infty}, v^{\infty}\right)$ is an equilibrium pair of (1.1). An equilibrium pair $\left(x^{\mathrm{e}}, v^{\mathrm{e}}\right)$ is said to be globally asymptotically stable (GAS), if $x^{\mathrm{e}}$ is a globally asymptotically stable equilibrium of (2.1). Obviously, if $(0,0)$ is an equilibrium pair of $(1.1)$, then $(0,0)$ is GAS if, and only, if the equilibrium 0 of the uncontrolled Lur'e system (1.1) is GAS. We say that an equilibrium pair $\left(x^{\mathrm{e}}, v^{\mathrm{e}}\right)$ of (1.1) is input-to-state stable (ISS) if there exist $\psi \in \mathcal{K} \mathcal{L}$ and $\varphi \in \mathcal{K}$ such that, for every $x^{0} \in \mathbb{R}^{n}$, every $v \in L_{\text {loc }}^{\infty}\left(\mathbb{R}_{+}, \mathbb{R}^{n}\right)$, and all $t \geq$ 0

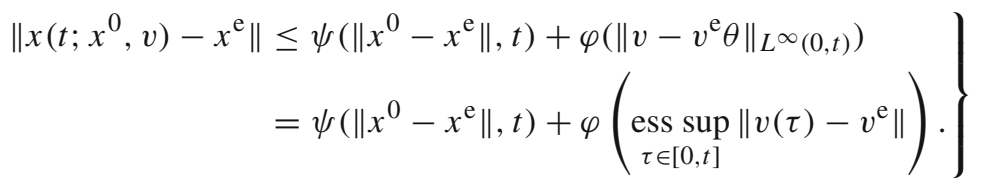

In the following, let $\mathbf{G}$ be the transfer function of the linear system specified by the triple $(A, B, C)$, that is, $\mathbf{G}(s)=C(s I-A)^{-1} B$, where $s$ is a complex variable. Applying output feedback of the form $u=K y+w$ to $(A, B, C)$, where $K \in \mathbb{R}^{m \times p}$ and $w$ is an input signal, leads to the closed-loop linear system specified by $(A+$ $B K C, B, C)$, the transfer function of which shall be denoted by $\mathbf{G}_{K}$. It is readily seen that

$$
\mathbf{G}_{K}(s)=C\left(s I-A_{K}\right)^{-1} B=C(s I-A-B K C)^{-1} B=\mathbf{G}(s)(I-K \mathbf{G}(s))^{-1},
$$

where, for notational convenience, we have set $A_{K}:=A+B K C$.

Let $\mathbb{S}_{\mathbb{C}}(A, B, C)$ denote the set of complex stabilizing output feedback gains for the linear system $(A, B, C)$, that is,

$$
\mathbb{S}_{\mathbb{C}}(A, B, C):=\left\{K \in \mathbb{C}^{m \times p}: A_{K} \text { is Hurwitz }\right\}
$$

Moreover, we define

$$
\mathbb{S}_{\mathbb{R}}(A, B, C):=\mathbb{S}_{\mathbb{C}}(A, B, C) \cap \mathbb{R}^{m \times p},
$$

the set of real stabilizing output feedback gains for $(A, B, C)$. Note that $\mathbb{S}_{\mathbb{R}}(A, B, C)$ and $\mathbb{S}_{\mathbb{C}}(A, B, C)$ may be empty. In principle, the Nyquist stability criterion can be used to check for non-emptiness of $\mathbb{S}_{\mathbb{R}}(A, B, C)$ or $\mathbb{S}_{\mathbb{C}}(A, B, C)$ : this is particularly straightforward in the case $m=p=1$. If $A$ is Hurwitz, then trivially $\mathbb{S}_{\mathbb{R}}(A, B, C)$ is non-empty. Furthermore, if $m=p,(A, B, C)$ is stabilizable and detectable and 
$\mathbf{G}+c I$ is positive real for some $c>0$, then $\mathbb{B}_{\mathbb{C}}(-\rho I, \rho) \subseteq \mathbb{S}_{\mathbb{C}}(A, B, C)$, where $\rho:=1 /(2 c)$.

The next theorem is a stability result for Lur'e systems that will be a key tool throughout this paper.

Theorem 2.1 Let $K \in \mathbb{S}_{\mathbb{R}}(A, B, C)$ and set $\gamma:=1 /\left\|\mathbf{G}_{K}\right\|_{H^{\infty}}$ (where $\gamma:=\infty$ if $\left.\left\|\mathbf{G}_{K}\right\|_{H^{\infty}}=0\right)$. The following statements hold.

1. If $\gamma<\infty$ and

$$
\|f(z)-K z\|<\gamma\|z\| \quad \forall z \in \mathbb{R}^{p}, z \neq 0,
$$

then the equilibrium 0 of the uncontrolled system (1.1) is GAS.

2. If $\gamma<\infty$ and there exists $\alpha \in \mathcal{K}_{\infty}$ such that

$$
\|f(z)-K z\| \leq \gamma\|z\|-\alpha(\|z\|) \quad \forall z \in \mathbb{R}^{p}
$$

then the equilibrium pair $(0,0)$ of (1.1) is ISS.

3. If $\gamma=\infty$, then the conclusions in statements (1) and (2) hold for every locally Lipschitz $f: \mathbb{R}^{p} \rightarrow \mathbb{R}^{m}$ such that $f(0)=0$.

Statements (1) and (2) are consequences results in [17,18] and [36], respectively. The scenario considered in statement (3), wherein $\left\|\mathbf{G}_{K}\right\|_{H^{\infty}}=0$ (or, equivalently, $\left.\mathbf{G}_{K}(s) \equiv 0\right)$, is not very interesting, but is included for mathematical completeness. Note that $\left\|\mathbf{G}_{K}\right\|_{H^{\infty}}=0$ if, and only if, $\|\mathbf{G}\|_{H^{\infty}}=0$. Consequently, if $(A, B)$ is controllable $((C, A)$ is observable $)$ and $C \neq 0(B \neq 0)$, then $\left\|\mathbf{G}_{K}\right\|_{H^{\infty}} \neq 0$. A proof of Theorem 2.1 can be found in the Appendix.

The following proposition is a special case of a well-known result from ISS theory.

Proposition 2.2 Assume that (0, 0) is an ISS equilibrium pair of (1.1). Then (1.1) has the 0-converging-input converging-state property: for every $x^{0} \in \mathbb{R}^{n}$ and for every $v \in L^{\infty}\left(\mathbb{R}_{+}, \mathbb{R}^{n}\right)$ such that $v(t) \rightarrow 0$ as $t \rightarrow \infty$, we have that $x\left(t ; x^{0}, v\right) \rightarrow 0$ as $t \rightarrow \infty$.

We emphasize that ISS is not a necessary condition for (1.1) to have the 0converging-input converging-state property (0-CICS property), see Example 4.5 further below. We now introduce a concept which strengthens the notion of the 0CICS property and is the primary focus of the present paper.

Definition 2.3 We say that (1.1) has the converging-input converging-state property (CICS property) if, for every $v^{\infty} \in \mathbb{R}^{n}$, there exists $x^{\infty} \in \mathbb{R}^{n}$ such that, for all $x^{0} \in \mathbb{R}^{n}$ 
and all $v \in L^{\infty}\left(\mathbb{R}_{+}, \mathbb{R}^{n}\right)$ with $\lim _{t \rightarrow \infty} v(t)=v^{\infty}, 1$

$$
\lim _{t \rightarrow \infty} x\left(t ; x^{0}, v\right)=x^{\infty}
$$

If (1.1) has the CICS property and if $f(0)=0$ (that is, the origin is an equilibrium of the uncontrolled Lur'e system (1.1)), then clearly (1.1) has the 0-CICS property.

The CICS property enables us to define steady-state gains for the Lur'e system (1.1). Indeed, assuming that (1.1) has the CICS property, the map

$$
\Gamma_{\text {is }}: \mathbb{R}^{n} \rightarrow \mathbb{R}^{n}, \quad v^{\infty} \mapsto x^{\infty}
$$

is well defined and is said to be the input-to-state steady-state gain (ISSS gain). The map

$$
\Gamma_{\text {io }}: \mathbb{R}^{n} \rightarrow \mathbb{R}^{p}, \quad v^{\infty} \mapsto C \Gamma_{\text {is }}\left(v^{\infty}\right)=C x^{\infty}
$$

is said to be the input-to-output steady-state gain (IOSS gain). In particular, if (1.1) has the CICS property, then, for every $v^{\infty}$, the point $x^{\infty}:=\Gamma_{\text {is }}\left(v^{\infty}\right)$ is a globally attractive equilibrium of the system

$$
\dot{x}(t)=A x(t)+B f(C x(t))+v^{\infty} \theta(t) \quad x(0)=x^{0} \quad t \geq 0 .
$$

\section{A necessary condition for CICS}

In this short section, we derive a necessary condition for the CICS property. In the following, the map

$$
F_{K}: \mathbb{R}^{p} \rightarrow \mathbb{R}^{p}, \quad z \mapsto z-\mathbf{G}_{K}(0)(f(z)-K z),
$$

where $K \in \mathbb{S}_{\mathbb{R}}(A, B, C)$, will play a key role. For a set $W \subseteq \mathbb{R}^{p}$, we shall denote the preimage of $W$ under $F_{K}$ by $F_{K}^{-1}(W)$. For $w \in \mathbb{R}^{p}$, it is convenient to set $F_{K}^{-1}(w):=$ $F_{K}^{-1}(\{w\})$. We note two simple, but important, properties of $F_{K}$ :

$$
F_{K}(\operatorname{im} C) \subseteq \operatorname{im} C, \quad F_{K}^{-1}(\operatorname{im} C) \subseteq \operatorname{im} C,
$$

a proof of which is contained in the Appendix. The next proposition describes properties of the map $F_{K}$ and shows how $F_{K}$ relates to equilibrium pairs $\left(x^{\infty}, v^{\infty}\right)$ of (1.1).

\footnotetext{
1 As an element in $L^{\infty}\left(\mathbb{R}_{+}, \mathbb{R}^{n}\right)$, strictly speaking, $v$ is not a function, but an equivalence class of functions. Therefore, we should clarify what we mean by

$$
\lim _{t \rightarrow \infty} v(t)=v^{\infty}
$$

We say that $(*)$ holds, if $\left\|v-v^{\infty} \theta\right\|_{L^{\infty}(t, \infty)} \rightarrow 0$ as $t \rightarrow \infty$, or equivalently, if there exists a representative $w$ in the equivalence class $v$ such that $w(t) \rightarrow v^{\infty}$ as $t \rightarrow \infty$.
} 
Proposition 3.1 Assume that $K \in \mathbb{S}_{\mathbb{R}}(A, B, C)$.

1. Let $v \in L^{\infty}\left(\mathbb{R}_{+}, \mathbb{R}^{n}\right)$ have a limit $v^{\infty}:=\lim _{t \rightarrow \infty} v(t)$ and assume that, for some $x^{0} \in \mathbb{R}^{n}$, the limit $x^{\infty}:=\lim _{t \rightarrow \infty} x\left(t ; x^{0}, v\right)$ exists. Then $\left(x^{\infty}, v^{\infty}\right)$ is an equilibrium pair of (1.1),

$$
x^{\infty}=-A_{K}^{-1}\left(B\left(f\left(C x^{\infty}\right)-K C x^{\infty}\right)+v^{\infty}\right)
$$

where $A_{K}:=A+B K C$, and $F_{K}\left(C x^{\infty}\right)=-C A_{K}^{-1} v^{\infty}$.

2. Let $v^{\infty} \in \mathbb{R}^{n}$ and assume that there exists $x^{\infty} \in \mathbb{R}^{n}$ such that, for all $x^{0} \in$ $\mathbb{R}^{n}, x\left(t ; x^{0}, v^{\infty} \theta\right) \rightarrow x^{\infty}$ as $t \rightarrow \infty$. Then $\# F_{K}^{-1}\left(-C A_{K}^{-1} v^{\infty}\right)=1$.

3. Let $v^{\infty} \in \mathbb{R}^{n}, y^{\infty} \in F_{K}^{-1}\left(-C A_{K}^{-1} v^{\infty}\right)$ and set $x^{\infty}:=-A_{K}^{-1}\left(B\left(f\left(y^{\infty}\right)-\right.\right.$ $\left.\left.K y^{\infty}\right)+v^{\infty}\right)$. Then $C x^{\infty}=y^{\infty}$ and $\left(x^{\infty}, v^{\infty}\right)$ is an equilibrium pair of (1.1).

Proof of Proposition 3.1. To prove statement (1), set $x(t):=x\left(t ; x^{0}, v\right)$ and note that $x$ satisfies

$$
\dot{x}(t)=A_{K} x(t)+B(f(C x(t))-K C x(t))+v(t) \text { for a.e. } t \geq 0 .
$$

Since $A_{K}$ is Hurwitz, it follows immediately that (3.2) holds. As an immediate consequence of (3.2), we have

$$
0=A_{K} x^{\infty}+B\left(f\left(C x^{\infty}\right)-K C x^{\infty}\right)+v^{\infty}=A x^{\infty}+B f\left(C x^{\infty}\right)+v^{\infty},
$$

showing that $\left(x^{\infty}, v^{\infty}\right)$ is an equilibrium pair of (1.1). Furthermore, applying $C$ to both sides of (3.2) and rearranging shows that $F_{K}\left(C x^{\infty}\right)=-C A_{K}^{-1} v^{\infty}$.

We proceed to prove statement (2). By statement (1), $x^{\infty}$ satisfies (3.2), and $C x^{\infty} \in F_{K}^{-1}\left(-C A_{K}^{-1} v^{\infty}\right)$, showing that $F_{K}^{-1}\left(-C A_{K}^{-1} v^{\infty}\right) \neq \varnothing$. Let $y_{1}, y_{2} \in$ $F_{K}^{-1}\left(-C A_{K}^{-1} v^{\infty}\right)$. It remains to show that $y_{1}=y_{2}$. To this end, set

$$
\xi_{i}:=-A_{K}^{-1}\left(B\left(f\left(y_{i}\right)-K y_{i}\right)+v^{\infty}\right), \quad i \in\{1,2\} .
$$

Then

$$
F_{K}\left(y_{i}\right)=y_{i}-\mathbf{G}_{K}(0)\left(f\left(y_{i}\right)-K y_{i}\right)=y_{i}-C \xi_{i}-C A_{K}^{-1} v^{\infty}, \quad i \in\{1,2\},
$$

But $F_{K}\left(y_{i}\right)=-C A_{K}^{-1} v^{\infty}$ for $i \in\{1,2\}$ and so, $y_{i}=C \xi_{i}$ for $i \in\{1,2\}$. Consequently, by (3.3),

$$
A_{K} \xi_{i}+B\left(f\left(C \xi_{i}\right)-K C \xi_{i}\right)+v^{\infty}=0, \quad i \in\{1,2\},
$$

and so

$$
A \xi_{i}+B f\left(C \xi_{i}\right)+v^{\infty}=0, \quad i \in\{1,2\},
$$


showing that $\left(\xi_{1}, v^{\infty}\right)$ and $\left(\xi_{2}, v^{\infty}\right)$ are equilibrium pairs of (1.1). Hence, $x\left(t ; \xi_{i}, v^{\infty} \theta\right)$ $=\xi_{i}$ for all $t \geq 0$ and it follows from the hypothesis that $\xi_{1}=x^{\infty}=\xi_{2}$. Thus, $y_{1}=C \xi_{1}=C \xi_{2}=y_{2}$, completing the proof.

To prove statement (3), note that

$$
C x^{\infty}=\mathbf{G}_{K}(0)\left(f\left(y^{\infty}\right)-K y^{\infty}\right)-C A_{K}^{-1} v^{\infty}=y^{\infty}-F_{K}\left(y^{\infty}\right)-C A_{K}^{-1} v^{\infty}=y^{\infty} .
$$

Therefore,

$$
A x^{\infty}+B f\left(C x^{\infty}\right)+v^{\infty}=A_{K} x^{\infty}+B\left(f\left(y^{\infty}\right)-K y^{\infty}\right)+v^{\infty}=0,
$$

showing that $\left(x^{\infty}, v^{\infty}\right)$ is an equilibrium pair of (1.1).

The following consequence of Proposition 3.1 provides, in terms of $F_{K}$, a necessary condition for the CICS property to hold.

Corollary 3.2 Let $K \in \mathbb{S}_{\mathbb{R}}(A, B, C)$. If the Lur'e system (1.1) has the CICS property, then $\# F_{K}^{-1}(z)=1$ for all $z \in \operatorname{im} C$.

It follows from (3.1) and Corollary 3.2 that, if (1.1) has the CICS property, then the restriction of $F_{K}$ to im $C$ provides a bijection from the subspace im $C$ into itself.

Proof of Corollary 3.2. Let $z \in \operatorname{im} C$. Then there exists $v^{\infty} \in \mathbb{R}^{n}$ such that $z=$ $-C A_{K}^{-1} v^{\infty}$. By the CICS property, it is clear that there exists $x^{\infty} \in \mathbb{R}^{n}$, such that for all $x^{0} \in \mathbb{R}^{n}, x\left(t ; x^{0}, v^{\infty} \theta\right) \rightarrow x^{\infty}$ as $t \rightarrow \infty$. Hence, by statement (2) of Proposition 3.1, $\# F_{K}^{-1}(z)=\# F_{K}^{-1}\left(-C A_{K}^{-1} v^{\infty}\right)=1$.

\section{Sufficient conditions for CICS}

In this section, we provide conditions which ensure that the Lur'e system (1.1) has the CICS property. The main result is Theorem 4.3 which, in turn, yields a host of sufficient conditions for the CICS property, formulated as Corollaries 4.8, 4.10, 4.11, 4.15 and 4.16 .

The next result provides conditions which guarantee certain surjectivity and injectivity properties of the map $F_{K}$. We denote the restriction of $F_{K}$ to im $C$ by $\hat{F}_{K}$. It follows from (3.1) that $\hat{F}_{K}$ maps into im $C$ and we define the co-domain of $\hat{F}_{K}$ to be equal to im $C$.

Proposition 4.1 Let $Y \subseteq \operatorname{im} C$ be nonempty, $K \in \mathbb{S}_{\mathbb{R}}(A, B, C)$, set $\gamma:=$ $1 /\left\|\mathbf{G}_{K}\right\|_{H^{\infty}}$ (where $\gamma:=\infty$ if $\left\|\mathbf{G}_{K}\right\|_{H^{\infty}}=0$ ) and assume that $f$ satisfies the condition:

(A) $\|f(z+\xi)-f(\xi)-K z\|<\gamma\|z\|$ for all $\xi \in Y$ and all $z \in \mathbb{R}^{p}, z \neq 0$.

The following statements hold.

1. $\# F_{K}^{-1}(z)=1$ for every $z \in \operatorname{im} C$ such that $F_{K}^{-1}(z) \cap Y \neq \emptyset$.

2. If

$$
\left\|\mathbf{G}_{K}(0)\right\|<\left\|\mathbf{G}_{K}\right\|_{H^{\infty}}
$$

then $F_{K}$ is surjective. 
3. If there exists $\zeta \in \mathbb{R}^{p}$ such that

$$
\gamma\|z\|-\|f(z+\zeta)-f(\zeta)-K z\| \rightarrow \infty \text { as }\|z\| \rightarrow \infty,
$$

then $F_{K}$ is surjective.

4. If $Y=\operatorname{im} C$ and (4.1) or (4.2) hold, then $\hat{F}_{K}$ is bijective.

Before we prove Proposition 4.1, we state and prove a simple lemma which will be a convenient tool in the following. In particular, it will be useful in the proof of Proposition 4.1.

Lemma 4.2 Let $g: \mathbb{R}^{p} \rightarrow \mathbb{R}^{m}$ be an arbitrary function and let $r>0$.

1. If there exists $\zeta \in \mathbb{R}^{p}$ such that

$$
r\|z\|-\|g(z+\zeta)-g(\zeta)\| \rightarrow \infty \text { as }\|z\| \rightarrow \infty,
$$

then, for every $\xi \in \mathbb{R}^{p}$,

$$
r\|z\|-\|g(z+\xi)-g(\xi)\| \rightarrow \infty \text { as }\|z\| \rightarrow \infty .
$$

2. If $g$ is continuous, $\|g(z)\|<r\|z\|$ for all non-zero $z \in \mathbb{R}^{p}$ and $r\|z\|-\|g(z)\| \rightarrow \infty$ as $\|z\| \rightarrow \infty$, then there exists $\alpha \in \mathcal{K}_{\infty}$ such that $\|g(z)\| \leq r\|z\|-\alpha(\|z\|)$ for all $z \in \mathbb{R}^{p}$.

Proof To prove statement (1), let $\xi \in \mathbb{R}^{p}$, set $w:=z+\xi-\zeta$ and note that $r\|z\|-\|g(z+\xi)-g(\xi)\|=r\|w+\zeta-\xi\|-\|g(w+\zeta)-g(\zeta)+g(\zeta)-g(\xi)\|$.

Consequently,

$$
\begin{aligned}
r\|z\|-\|g(z+\xi)-g(\xi)\| \geq & r\|w\|-\|g(w+\zeta)-g(\zeta)\|-r\|\zeta-\xi\| \\
& -\|g(\zeta)-g(\xi)\|,
\end{aligned}
$$

and since $\|w\| \rightarrow \infty$ as $\|z\| \rightarrow \infty$, the claim follows from (4.3).

To prove statement (2), define $\beta: \mathbb{R}_{+} \rightarrow \mathbb{R}_{+}$by

$$
\beta(s):=\inf _{\|z\| \geq s}(r\|z\|-\|g(z)\|) \quad s \geq 0 .
$$

Then $\beta$ is continuous (by the continuity of $g$ ), $\beta(0)=0, \beta(s)>0$ for $s>0, \beta$ is nondecreasing, $\beta(s) \rightarrow \infty$ as $s \rightarrow \infty$ and $\|g(z)\| \leq r\|z\|-\beta(\|z\|)$ for all $z \in \mathbb{R}^{p}$. Therefore, setting $\alpha(s):=\left(1-\mathrm{e}^{-s}\right) \beta(s)$, it is clear that $\alpha \in \mathcal{K}_{\infty}$ and $\|g(z)\| \leq r\|z\|-\alpha(\|z\|)$ for all $z \in \mathbb{R}^{p}$, completing the proof.

Proof of Proposition 4.1. If $\mathbf{G}_{K}(0)=0$, then $F_{K}(z)=z$ for all $z \in \mathbb{R}^{p}$. Consequently, the maps $F_{K}$ and $\hat{F}_{K}$ are bijective and there is nothing to prove. Let us now assume that $\mathbf{G}_{K}(0) \neq 0$. Then, $\left\|\mathbf{G}_{K}\right\|_{H^{\infty}} \neq 0$, and so, $0<\gamma<\infty$. 
To prove statement (1), let $z \in \operatorname{im} C$ and assume that $F_{K}^{-1}(z) \cap Y \neq \emptyset$. Let $\xi_{1} \in F_{K}^{-1}(z) \cap Y$ and $\xi_{2} \in F_{K}^{-1}(z)$. To establish that $\# F_{K}^{-1}(z)=1$, it suffices to show that $\xi_{1}=\xi_{2}$. Since $F_{K}\left(\xi_{1}\right)=F_{K}\left(\xi_{2}\right)$, it follows that

$$
\left\|\xi_{2}-\xi_{1}\right\|=\left\|\mathbf{G}_{K}(0)\left(f\left(\xi_{2}\right)-f\left(\xi_{1}\right)-K\left(\xi_{2}-\xi_{1}\right)\right)\right\| .
$$

If $\xi_{1} \neq \xi_{2}$, then, by condition (A),

$$
\left\|\xi_{2}-\xi_{1}\right\|<\left\|\mathbf{G}_{K}(0)\right\| \gamma\left\|\xi_{2}-\xi_{1}\right\| \leq\left\|\xi_{2}-\xi_{1}\right\|
$$

which is impossible. Hence, $\xi_{1}=\xi_{2}$.

We proceed to prove statement (2). To show surjectivity of $F_{K}$, note that, by [32, Theorem 9.36], it is sufficient to prove that $F_{K}$ is coercive, that is,

$$
\frac{1}{\|z\|}\left\langle F_{K}(z), z\right\rangle \rightarrow \infty \text { as }\|z\| \rightarrow \infty
$$

To establish (4.4), we note that, for all $z \in \mathbb{R}^{p}$,

$$
\begin{aligned}
\frac{1}{\|z\|}\left\langle F_{K}(z), z\right\rangle= & \|z\|+\frac{1}{\|z\|}\left\langle\mathbf{G}_{K}(0)(f(z)-K z), z\right\rangle \geq\|z\| \\
& -\left\|\mathbf{G}_{K}(0)\right\|\|f(z)-K z\|,
\end{aligned}
$$

and hence

$$
\frac{1}{\|z\|}\left\langle F_{K}(z), z\right\rangle \geq\|z\|-\left\|\mathbf{G}_{K}(0)\right\|(\|f(z)-f(\xi)-K z\|+\|f(\xi)\|) \quad \forall z \in \mathbb{R}^{p},
$$

where $\xi \in Y$. By condition (A),

$$
\|f(z+\xi)-f(\xi)-K z\| \leq \gamma\|z\| \quad \forall z \in \mathbb{R}^{p}
$$

Consequently, for all $z \in \mathbb{R}^{p}$,

$$
\begin{aligned}
\|f(z)-f(\xi)-K z\| & \leq\|f(z-\xi+\xi)-f(\xi)-K(z-\xi)\|+\|K \xi\| \\
& \leq \gamma\|z-\xi\|+\|K \xi\|,
\end{aligned}
$$

and thus,

$$
\|f(z)-f(\xi)-K z\| \leq \gamma\|z\|+(\|K\|+\gamma)\|\xi\| \quad \forall z \in \mathbb{R}^{p}
$$

\section{Setting}

$$
\kappa:=\left\|\mathbf{G}_{K}(0)\right\|(\|f(\xi)\|+(\|K\|+\gamma)\|\xi\|),
$$


and invoking (4.5) and (4.6), we conclude that

$$
\frac{1}{\|z\|}\left\langle F_{K}(z), z\right\rangle \geq\left(1-\gamma\left\|\mathbf{G}_{K}(0)\right\|\right)\|z\|-\kappa \quad \forall z \in \mathbb{R}^{p}
$$

Now, by hypothesis, $\left\|\mathbf{G}_{K}(0)\right\|<\left\|\mathbf{G}_{K}\right\|_{H^{\infty}}$, or, equivalently, $1-\gamma\left\|\mathbf{G}_{K}(0)\right\|>0$, implying that (4.4) holds, and so surjectivity of $F_{K}$ follows.

To prove statement (3), let $\xi \in Y$. By hypothesis and statement (1) of Lemma 4.2 (applied to $g(z)=f(z)-K z)$,

$$
\gamma\|z\|-\|f(z+\xi)-f(\xi)-K z\| \rightarrow \infty \quad \text { as }\|z\| \rightarrow \infty
$$

Together with assumption (A) and an application of statement (2) of Lemma 4.2 this shows that there exists $\alpha \in \mathcal{K}_{\infty}$ such that

$$
\|f(z+\xi)-f(\xi)-K z\| \leq \gamma\|z\|-\alpha(\|z\|) \quad \forall z \in \mathbb{R}^{p}
$$

An argument very similar to that leading to (4.6) yields

$$
\|f(z)-f(\xi)-K z\| \leq \gamma\|z\|-\alpha(\|z-\xi\|)+(\|K\|+\gamma)\|\xi\| \quad \forall z \in \mathbb{R}^{p},
$$

Together with (4.5) this implies

$$
\frac{1}{\|z\|}\left\langle F_{K}(z), z\right\rangle \geq\left(1-\gamma\left\|\mathbf{G}_{K}(0)\right\|\right)\|z\|+\left\|\mathbf{G}_{K}(0)\right\| \alpha(\|z-\xi\|)-\kappa \quad \forall z \in \mathbb{R}^{p},
$$

with $\kappa$ defined by (4.7). Now $1-\gamma\left\|\mathbf{G}_{K}(0)\right\| \geq 1-\gamma\left\|\mathbf{G}_{K}\right\|_{H^{\infty}}=0$ and (4.4) follows, showing that $F_{K}$ is coercive and hence surjective.

Finally, to prove statement (4), assume that $Y=\operatorname{im~} C$ and that (4.1) or (4.2) are satisfied. Then the map $F_{K}$ is surjective (as follows from statement (2) if (4.1) holds and from statement (3) if (4.2) holds). Surjectivity of $F_{K},(3.1)$ and statement (1) guarantee that $\# F_{K}^{-1}(z)=1$ for all $z \in \operatorname{im} C$. Writing $F_{K}^{-1}(z)=\left\{y_{z}\right\}$ for every $z \in \operatorname{im} C$ and, once again, invoking (3.1), we conclude that $y_{z} \in \operatorname{im} C$ and bijectivity of $\hat{F}_{K}$ follows.

For $\tau \geq 0$, we define the left-shift operator $\Lambda_{\tau}$ by $\left(\Lambda_{\tau} v\right)(t)=v(t+\tau)$ for all $t \geq 0$, where $v$ is an arbitrary function $\mathbb{R}_{+} \rightarrow \mathbb{R}^{n}$. A subset $\mathcal{V} \subseteq L^{\infty}\left(\mathbb{R}_{+}, \mathbb{R}^{n}\right)$ is said to be equi-convergent to $v^{\infty} \in \mathbb{R}^{n}$ if, for every $\varepsilon>0$, there exists $\tau \geq 0$ such that

$$
\left\|\Lambda_{\tau} v-v^{\infty} \theta\right\|_{L^{\infty}} \leq \varepsilon \quad \forall v \in \mathcal{V}
$$

The following theorem is the main result of this section.

Theorem 4.3 Let $Y \subseteq \operatorname{im} C$ be nonempty, $K \in \mathbb{S}_{\mathbb{R}}(A, B, C), v^{\infty} \in \mathbb{R}^{n}$ and set $\gamma:=1 /\left\|\mathbf{G}_{K}\right\|_{H^{\infty}}$ (where $\gamma:=\infty$ if $\left\|\mathbf{G}_{K}\right\|_{H^{\infty}}=0$ ). Assume that condition (A) 
holds and that $F_{K}^{-1}\left(-C A_{K}^{-1} v^{\infty}\right) \cap Y \neq \emptyset$. Then $\# F_{K}^{-1}\left(-C A_{K}^{-1} v^{\infty}\right)=1$ and, writing $\left\{y^{\infty}\right\}=F_{K}^{-1}\left(-C A_{K}^{-1} v^{\infty}\right)$, the pair $\left(x^{\infty}, v^{\infty}\right)$, where

$$
x^{\infty}:=-A_{K}^{-1}\left(B\left(f\left(y^{\infty}\right)-K y^{\infty}\right)+v^{\infty}\right),
$$

is an equilibrium pair of system (1.1). Furthermore, $C x^{\infty}=y^{\infty}$ and the following statements hold.

1. The equilibrium pair $\left(x^{\infty}, v^{\infty}\right)$ is GAS, and, for every $x^{0} \in \mathbb{R}^{n}$ and every $v \in$ $L^{\infty}\left(\mathbb{R}_{+}, \mathbb{R}^{n}\right)$ such that $\lim _{t \rightarrow \infty} v(t)=v^{\infty}$, we have that either $x\left(t ; x^{0}, v\right) \rightarrow x^{\infty}$ or $\left\|x\left(t ; x^{0}, v\right)\right\| \rightarrow \infty$ as $t \rightarrow \infty$.

2. Under the additional assumption that, for some $\zeta \in \mathbb{R}^{p}$,

$$
\gamma\|z\|-\|f(z+\zeta)-f(\zeta)-K z\| \rightarrow \infty \text { as }\|z\| \rightarrow \infty
$$

$\left(x^{\infty}, v^{\infty}\right)$, with $x^{\infty}$ given by (4.8), is an ISS equilibrium pair of (1.1) and there exist $\psi_{1}, \psi_{2} \in \mathcal{K} \mathcal{L}$ and $\varphi \in \mathcal{K}$ such that, for all $\left(x^{0}, v\right) \in \mathbb{R}^{n} \times L^{\infty}\left(\mathbb{R}_{+}, \mathbb{R}^{n}\right)$ and all $t \geq 0$,

$$
\begin{aligned}
\left\|x\left(t ; x^{0}, v\right)-x^{\infty}\right\| \leq & \psi_{1}\left(\left\|x^{0}-x^{\infty}\right\|, t\right)+\psi_{2}\left(\left\|v-v^{\infty} \theta\right\|_{L^{\infty}, t}\right) \\
& +\varphi\left(\left\|\Lambda_{t / 2}\left(v-v^{\infty} \theta\right)\right\|_{L^{\infty}}\right) .
\end{aligned}
$$

In particular, for every $x^{0} \in \mathbb{R}^{n}$ and every $v \in L^{\infty}\left(\mathbb{R}_{+}, \mathbb{R}^{n}\right)$ such that $\lim _{t \rightarrow \infty} v(t)=$ $v^{\infty}$

$$
\lim _{t \rightarrow \infty} x\left(t ; x^{0}, v\right)=x^{\infty}
$$

and the convergence is uniform in the following sense: given a set of inputs $\mathcal{V} \subseteq$ $L^{\infty}\left(\mathbb{R}_{+}, \mathbb{R}^{n}\right)$ which is equi-convergent to $v^{\infty}$ and $\kappa>0$, then the set of solutions

$$
\left\{x\left(\cdot ; x^{0}, v\right):\left(x^{0}, v\right) \in \mathbb{R}^{n} \times \mathcal{V} \text { such that }\left\|x^{0}\right\|+\|v\|_{L^{\infty}} \leq \kappa\right\}
$$

is equi-convergent to $x^{\infty}$.

Before proving Theorem 4.3, we provide some commentary.

Remark 4.4 (a) Assumption (A) is an incremental condition which is weaker than a global Lipschitz condition, since (A) only needs to hold for all $\xi \in Y$. We mention that the number $1 /\left\|\mathbf{G}_{K}\right\|_{H^{\infty}}$ appearing on the right-hand side of the inequality in assumption (A) is equal to the structured complex stability radius of the Hurwitz $A+B K C$ with respect to the perturbation structure given by $B$ and $C$, see [16].

(b) The set $Y \subseteq \operatorname{im} C$ should be interpreted as the set of all "achievable" output limits. Indeed, if $K \in \mathbb{S}_{\mathbb{R}}(A, B, C)$, condition (A) is satisfied and there exists $\zeta$ such that (4.9) holds, then, for every $y^{\infty} \in Y$, there exists $v^{\infty} \in \mathbb{R}^{n}$ such that $F_{K}\left(y^{\infty}\right)=-C A_{K}^{-1} v^{\infty}$ and, by Theorem 4.3, $C x\left(t ; x^{0}, v\right) \rightarrow y^{\infty}$ as $t \rightarrow \infty$ for every $x^{0}$ and every $v$ convergent to $v^{\infty}$. 
(c) It may appear that $y^{\infty}$ and $x^{\infty}$ depend on $K$, but this is not the case. Indeed, let non-empty $Y \subseteq \operatorname{im} C$ and $v^{\infty} \in \mathbb{R}^{n}$ be given, let $K_{1}, K_{2} \in \mathbb{S}_{\mathbb{R}}(A, B, C)$ and set $\gamma_{i}:=1 /\left\|\mathbf{G}_{K_{i}}\right\|_{H^{\infty}}$, where $i=1,2$. Assume that condition (A) holds with $K=K_{i}$ and $\gamma=\gamma_{i}$ and $F_{K_{i}}^{-1}\left(-C A_{K_{i}}^{-1} v^{\infty}\right) \cap Y \neq \emptyset$ for $i=1,2$. By Theorem 4.3, the pre-image $F_{K_{i}}^{-1}\left(-C A_{K_{i}}^{-1} v^{\infty}\right)$ is a singleton, the element of which we denote by $y_{i}^{\infty}$. Defining, as in (4.8),

$$
x_{i}^{\infty}:=-A_{K_{i}}^{-1}\left(B\left(f\left(y_{i}^{\infty}\right)-K_{i} y_{i}^{\infty}\right)+v^{\infty}\right), \quad i=1,2,
$$

it follows from Theorem 4.3 that $\left(x_{1}^{\infty}, v^{\infty}\right)$ and $\left(x_{2}^{\infty}, v^{\infty}\right)$ are globally asymptotically stable equilibrium pairs, which obviously implies that $x_{1}^{\infty}=x_{2}^{\infty}$. Consequently, $y_{1}^{\infty}=C x_{1}^{\infty}=C x_{2}^{\infty}=y_{2}^{\infty}$. In particular, if $Y=\operatorname{im} C$ and there exists $\zeta_{i} \in \mathbb{R}^{p}$ such that (4.9) holds with $K=K_{i}$ and $\zeta=\zeta_{i}$ for $i=1,2$, the map $\hat{F}_{K_{i}}$ is bijective for $i=1,2$ (by Proposition 4.1) and $\hat{F}_{K_{1}}^{-1}\left(C A_{K_{1}}^{-1} w\right)=\hat{F}_{K_{2}}^{-1}\left(C A_{K_{2}}^{-1} w\right)$ for all $w \in \mathbb{R}^{n}$.

(d) A (conservative) sufficient condition for (4.9) is that there exists $\rho \in(0, \gamma)$ and $\zeta \in \mathbb{R}^{p}$ such that

$$
\|f(z+\zeta)-f(\zeta)-K z\| \leq \rho\|z\| \quad \forall z \in \mathbb{R}^{p}
$$

We give a simple example which satisfies (4.9), but for which there does not exist $\rho \in(0, \gamma)$ such that (4.11) holds. Consider $p=m=\gamma=1, K=0$ and

$$
f: \mathbb{R}_{+} \rightarrow \mathbb{R}_{+}, \quad z \mapsto 0.9 z+0.1(z \sin z-\ln (1+z)),
$$

and note that

$$
|z|-|g(z)|=0.1 z(1-\sin z)+0.1 \ln (1+z) \geq 0.1 \ln (1+z) \quad \forall z \in \mathbb{R} .
$$

Consequently, the divergence condition (4.9) holds (as does condition (A)), but there does not exist $\rho \in(0,1)$ such that (4.11) holds.

(e) The estimate (4.10) is the result of a suitable modification of an ISS estimate (see the proof below for details). The three terms on the right-hand side, all of which converge to 0 as $t \rightarrow \infty$, relate, respectively, to the initial "error" $x^{0}-x^{\infty}$, the $L^{\infty}$-norm of $v-v^{\infty} \theta$ over the entire time interval $[0, \infty)$ and the $L^{\infty}$-norm of $v-v^{\infty} \theta$ over $[t / 2, \infty$ ) (the latter converging to 0 as $t \rightarrow \infty$ ). Note that the $\mathcal{K} \mathcal{L}$-functions $\psi_{1}$ and $\psi_{2}$ and the $\mathcal{K}$-function $\varphi$ only depend on $v^{\infty}$ and $x^{\infty}$ and hence (4.10) is uniform in $x^{0}$ and $v$. Furthermore, (4.10) evidently implies CICS and the equi-convergence property formulated towards the end of statement (2) of Theorem 4.3.

Proof of Theorem 4.3. By hypothesis, assumption (A) holds and $F_{K}^{-1}\left(-C A_{K}^{-1} v^{\infty}\right) \cap$ $Y \neq \emptyset$, and thus, statement (1) of Proposition 4.1 yields that $\# F_{K}^{-1}\left(-C A_{K}^{-1} v^{\infty}\right)=1$. For $x^{\infty}$ given by (4.8), it follows from statement (3) of Proposition 3.1 that $\left(x^{\infty}, v^{\infty}\right)$ 
is an equilibrium pair of (1.1) and $C x^{\infty}=y^{\infty}$. Define $\tilde{f}: \mathbb{R}^{p} \rightarrow \mathbb{R}^{m}$ by

$$
\tilde{f}(z):=f\left(z+y^{\infty}\right)-f\left(y^{\infty}\right) \quad \forall z \in \mathbb{R}^{p} .
$$

A straightforward calculation shows that

$$
A\left(z+x^{\infty}\right)+B f\left(C\left(z+x^{\infty}\right)\right)+v^{\infty}=A z+B \tilde{f}(C z) \quad \forall z \in \mathbb{R}^{n} .
$$

Moreover, since $y^{\infty} \in Y$, it follows from assumption (A) that

$$
\|\tilde{f}(z)-K z\|<\gamma\|z\| \quad \forall z \in \mathbb{R}^{p}, z \neq 0 .
$$

To prove statement (1), note that, by (4.12), a function $x$ satisfies

$$
\dot{x}(t)=A x(t)+B f(C x(t))+v^{\infty} \theta(t) \quad \forall t \geq 0,
$$

if, and only if, $\tilde{x}:=x-x^{\infty} \theta$ satisfies

$$
\dot{\tilde{x}}(t)=A \tilde{x}(t)+B \tilde{f}(C \tilde{x}(t)) \quad \forall t \geq 0
$$

Consequently, the equilibrium $x^{\infty}$ of (4.14) is GAS if, and only if, the equilibrium 0 of (4.15) is GAS. Invoking (4.13) in conjunction with statements (1) and (3) of Theorem 2.1 shows that the equilibrium 0 of (4.15) is GAS and hence, $x^{\infty}$ is a GAS equilibrium of system (4.14). An application of [41, Theorem 1] (or, alternatively, of [25, Theorem 4.3]) allows us to conclude that, for $x^{0} \in \mathbb{R}^{n}$ and $v \in L^{\infty}\left(\mathbb{R}_{+}, \mathbb{R}^{n}\right)$ with $v(t) \rightarrow v^{\infty}$ as $t \rightarrow \infty$, we have that either $x\left(t ; x^{0}, v\right) \rightarrow x^{\infty}$ or $\left\|x\left(t ; x^{0}, v\right)\right\| \rightarrow \infty$ as $t \rightarrow \infty$, completing the proof of statement (1).

We proceed to prove statement (2). To this end, let $v \in L_{\text {loc }}^{\infty}\left(\mathbb{R}_{+}, \mathbb{R}^{n}\right)$ and set $\tilde{v}=v-v^{\infty} \theta$. Invoking (4.12) shows that a function $x$ solves (1.1) if, and only if, $\tilde{x}:=x-x^{\infty} \theta$ solves

$$
\dot{\tilde{x}}(t)=A \tilde{x}(t)+B \tilde{f}(C \tilde{x}(t))+\tilde{v}(t) \text { for almost all } t \geq 0 .
$$

Consequently, the equilibrium pair $\left(x^{\infty}, v^{\infty}\right)$ of (1.1) is ISS if, and only if, the equilibrium pair $(0,0)$ of (4.16) is ISS. By (4.9) and statement (1) of Lemma 4.2,

$$
\gamma\|z\|-\|\tilde{f}(z)-K z\| \rightarrow \infty \quad \text { as }\|z\| \rightarrow \infty .
$$

This, together with (4.13) and statement (2) of Lemma 4.2, shows that there exists $\alpha \in \mathcal{K}_{\infty}$ such that $\|\tilde{f}(z)-K z\| \leq \gamma\|z\|-\alpha(\|z\|)$ for $z \in \mathbb{R}^{p}$. Statements (2) and (3) of Theorem 2.1 now show that $(0,0)$ is an ISS equilibrium pair of the system (4.16) and thus, the equilibrium pair $\left(x^{\infty}, v^{\infty}\right)$ of (1.1) is ISS. Consequently, there exist $\psi \in \mathcal{K} \mathcal{L}$ and $\varphi \in \mathcal{K}$ such that

$$
\begin{aligned}
\left\|x\left(t ; x^{0}, v\right)-x^{\infty}\right\| \leq \psi & \left(\left\|x^{0}-x^{\infty}\right\|, t\right)+\varphi\left(\left\|v-v^{\infty} \theta\right\|_{L^{\infty}(0, t)}\right) \\
& \forall x^{0} \in \mathbb{R}^{n}, \quad \forall v \in L_{\mathrm{loc}}^{\infty}\left(\mathbb{R}_{+}, \mathbb{R}^{n}\right), \quad \forall t \geq 0 .
\end{aligned}
$$


It remains to show that (4.10) holds. To this end, let $x^{0} \in \mathbb{R}^{n}$ and $v \in L^{\infty}\left(\mathbb{R}_{+}, \mathbb{R}^{n}\right)$ and note that, by the state transition property of system (1.1),

$$
x\left(t ; x^{0}, v\right)=x\left(t / 2 ; x\left(t / 2 ; x^{0}, v\right), \Lambda_{t / 2} v\right) \quad \forall t \geq 0 .
$$

Hence, by (4.17),

$$
\begin{aligned}
\left\|x\left(t ; x^{0}, v\right)-x^{\infty}\right\| \leq & \psi\left(\left\|x\left(t / 2 ; x^{0}, v\right)-x^{\infty}\right\|, t / 2\right) \\
& +\varphi\left(\left\|\Lambda_{t / 2} v-v^{\infty} \theta\right\|_{L^{\infty}}\right) \quad \forall t \geq 0 .
\end{aligned}
$$

Another application of (4.17) yields

$$
\begin{gathered}
\left\|x\left(t ; x^{0}, v\right)-x^{\infty}\right\| \leq \\
\psi\left(\psi\left(\left\|x^{0}-x^{\infty}\right\|, t / 2\right)+\varphi\left(\left\|v-v^{\infty} \theta\right\|_{L^{\infty}}\right), t / 2\right) \\
+\varphi\left(\left\|\Lambda_{t / 2}\left(v-v^{\infty} \theta\right)\right\|_{L^{\infty}}\right) \quad \forall t \geq 0 .
\end{gathered}
$$

Consequently, defining $\psi_{1}, \psi_{2} \in \mathcal{K} \mathcal{L}$ by

$$
\psi_{1}(s, t):=\psi(2 \psi(s, t / 2), t / 2) \quad \text { and } \quad \psi_{2}(s, t):=\psi(2 \varphi(s), t / 2) \quad \forall s, t \geq 0 \text {, }
$$

we obtain, for $t \geq 0$,

$$
\begin{aligned}
\left\|x\left(t ; x^{0}, v\right)-x^{\infty}\right\| \leq & \psi_{1}\left(\left\|x^{0}-x^{\infty}\right\|, t\right)+\psi_{2}\left(\left\|v-v^{\infty} \theta\right\|_{L^{\infty}, t}\right) \\
& +\varphi\left(\left\|\Lambda_{t / 2}\left(v-v^{\infty} \theta\right)\right\|_{L^{\infty}}\right),
\end{aligned}
$$

which is (4.10).

We illustrate the conclusions of Theorem 4.3 with some simple examples.

Example 4.5 Consider the one-dimensional Lur'e system

$$
\dot{x}(t)=-x(t)+f(x(t))+v(t) \quad t \geq 0 .
$$

Note that here $n=1, A=-1$ and $B=C=1$. We choose $K=0$ and so

$$
\mathbf{G}_{K}(s)=\mathbf{G}_{0}(s)=\mathbf{G}(s)=\frac{1}{s+1} .
$$

Since $\|\mathbf{G}\|_{H^{\infty}}=\mathbf{G}(0)=1$, we have $\gamma=1$.

(a) Let $f: \mathbb{R} \rightarrow \mathbb{R}$ be given by

$$
f(z)=z-\operatorname{sign}(z)\left(1-\mathrm{e}^{-|z|}\right) \quad \forall z \in \mathbb{R} .
$$

Since

$$
f^{\prime}(z)=1-\mathrm{e}^{-|z|} \forall z \in \mathbb{R},
$$


the mean-value theorem guarantees that

$$
|f(z+\xi)-f(\xi)|<|z| \quad \forall \xi, z \in \mathbb{R}, z \neq 0
$$

Furthermore,

$$
F_{0}(z)=z-f(z)=\operatorname{sign}(z)\left(1-\mathrm{e}^{-|z|}\right) \quad \forall z \in \mathbb{R},
$$

and so, $F_{0}(\mathbb{R})=(-1,1)$. Setting $Y:=\operatorname{im} C=\mathbb{R}$, we see that, for every $v^{\infty} \in(-1,1)$, the assumptions of statement (1) of Theorem 4.3 are satisfied. Therefore, if $v^{\infty} \in(-1,1)$, then, for all $x^{0} \in \mathbb{R}$ and all $v \in L^{\infty}\left(\mathbb{R}_{+}, \mathbb{R}\right)$ such that $\lim _{t \rightarrow \infty} v(t)=v^{\infty}$, we have that either $x\left(t ; x^{0}, v\right) \rightarrow x^{\infty}=F_{0}^{-1}\left(v^{\infty}\right)$ or $\left|x\left(t ; x^{0}, v\right)\right| \rightarrow \infty$ as $t \rightarrow \infty$. We show that divergence is not possible. Seeking a contradiction, suppose that there exist $v^{\infty} \in(-1,1), v \in L^{\infty}\left(\mathbb{R}_{+}, \mathbb{R}\right)$ with $\lim _{t \rightarrow \infty} v(t)=v^{\infty}$ and $x^{0} \in \mathbb{R}$ such that $\left|x\left(t ; x^{0}, v\right)\right| \rightarrow \infty$ as $t \rightarrow \infty$. Setting $x(t):=x\left(t ; x^{0}, v\right)$, we have that either $x(t) \rightarrow \infty$ or $x(t) \rightarrow-\infty$ as $t \rightarrow \infty$. If $x(t) \rightarrow \infty$ as $t \rightarrow \infty$, then there exists $\tau \geq 0$ such that

$$
\dot{x}(t)=-1+\mathrm{e}^{-x(t)}+v(t) \leq\left(v^{\infty}-1\right) / 2<0 \quad \forall t \geq \tau .
$$

But this implies that $x(t) \rightarrow-\infty$ as $t \rightarrow \infty$, providing the desired contradiction. Similarly, if $x(t) \rightarrow-\infty$ as $t \rightarrow \infty$, then there exists $\tau \geq 0$ such that

$$
\dot{x}(t)=1-\mathrm{e}^{x(t)}+v(t) \geq\left(v^{\infty}+1\right) / 2>0 \quad \forall t \geq \tau,
$$

showing that $x(t) \rightarrow \infty$ as $t \rightarrow \infty$, which is impossible.

The above analysis shows in particular that the system (4.18) has the 0-CICS property. Note that the equilibrium pair $(0,0)$ of $(4.18)$ is not ISS (since the input $v(t) \equiv 1+\varepsilon, \varepsilon>0$, produces an unbounded solution).

(b) Consider again system (4.18), but now with $f: \mathbb{R} \rightarrow \mathbb{R}$ given by

$$
f(z)=z-\operatorname{sat}(z) \mathrm{e}^{-|z|}, \quad \forall z \in \mathbb{R},
$$

where $\operatorname{sat}(z):=z$ for $|z| \leq 1$ and $\operatorname{sat}(z):=\operatorname{sign}(z)$ for $|z|>1$. Set $Y:=\{0\}$ and let $v^{\infty}=0$. Since,

$$
|f(z)|<|z| \quad \forall z \neq 0
$$

the assumptions of statement (1) of Theorem 4.3 are satisfied and it follows that $y^{\infty}=x^{\infty}=0$, the equilibrium 0 of the uncontrolled system (4.18) is GAS, and, for every $x^{0} \in \mathbb{R}$ and every $v \in L^{\infty}\left(\mathbb{R}_{+}, \mathbb{R}\right)$ with $\lim _{t \rightarrow \infty} v(t)=0$, either $x\left(t ; x^{0}, v\right) \rightarrow 0$ or $\left|x\left(t ; x^{0}, v\right)\right| \rightarrow \infty$ as $t \rightarrow \infty$. Divergence is possible: indeed, with $v$ given by $v(t)=2 /(t+e)$, it is straightforward to verify that $x(t ; 1, v)=$ $\ln (t+e)$. 
Example 4.6 Consider the two-dimensional Lur'e system

$$
\left.\begin{array}{l}
\dot{x}_{1}(t)=-x_{1}(t)+x_{2}(t)-f\left(2 x_{1}(t)+x_{2}(t)\right)+v_{1}(t) \\
\dot{x}_{2}(t)=-x_{1}(t)-3 x_{2}(t)+3 f\left(2 x_{1}(t)+x_{2}(t)\right)+v_{2}(t)
\end{array}\right\} \quad t \geq 0,
$$

with nonlinearity $f \in \mathcal{F}$, where $\mathcal{F}$ is the set of all of continuously differentiable functions $f: \mathbb{R} \rightarrow \mathbb{R}$ such that

$$
\left.\begin{array}{l}
f(0)=0, \quad f^{\prime}(z) \geq 0 \quad \forall z \in \mathbb{R}, \\
f^{\prime}(z) \leq 1 / 2 \quad \forall z \in \mathbb{R} \backslash(3,4) \quad \text { and } \quad \max _{y \in[3,4]} f^{\prime}(z)=2 .
\end{array}\right\}
$$

Setting

$$
A:=\left(\begin{array}{cc}
-1 & 1 \\
-1 & -3
\end{array}\right), \quad B:=\left(\begin{array}{c}
-1 \\
3
\end{array}\right), \quad C:=\left(\begin{array}{ll}
2 & 1
\end{array}\right)
$$

it is clear that system (4.20) is of the form (1.1). The matrix A is Hurwitz (-2 is an eigenvalue of algebraic multiplicity two) and the transfer function of the linear system $(A, B, C)$ is $\mathbf{G}(s)=(s+4) /(s+2)^{2}$. Choosing $K=0$, we have

$$
\left\|\mathbf{G}_{K}\right\|_{H^{\infty}}=\left\|\mathbf{G}_{0}\right\|_{H^{\infty}}=\|\mathbf{G}\|_{H^{\infty}}=\mathbf{G}(0)=1
$$

and thus, $\gamma=1$. It follows from (4.21) that $|z|-|f(z)| \rightarrow \infty$ as $|z| \rightarrow \infty$ and so, (4.9) holds with $\zeta=0$. Using elementary calculus, it is not difficult to show that, for every $\xi \in \mathbb{R} \backslash(1,6)$, there exists $a_{\xi} \in(0,1)$ such that

$$
|f(z+\xi)-f(\xi)| \leq a_{\xi}|z| \quad \forall z \in \mathbb{R} .
$$

Hence, condition (A) holds with $Y:=\mathbb{R} \backslash(1,6)$. Furthermore, $F_{0}(z)=F_{K}(z)=$ $z-f(z)$, and so, using (4.21),

$$
F_{0}(Y)=(-\infty, 1-f(1)] \cup[6-f(6), \infty) \supseteq(-\infty, 1 / 2] \cup[6, \infty) .
$$

According to statement (2) of Theorem 4.3 , for every $v^{\infty}=\left(v_{1}^{\infty}, v_{2}^{\infty}\right)^{\mathrm{T}} \in \mathbb{R}^{2}$ such that

$$
\left(5 v_{1}^{\infty}+3 v_{2}^{\infty}\right) / 4=-C A^{-1} v^{\infty} \in F_{0}(Y)
$$

there exists $x^{\infty} \in \mathbb{R}$ such that, for all $x^{0} \in \mathbb{R}$ and all $v \in L^{\infty}\left(\mathbb{R}_{+}, \mathbb{R}\right)$ with $\lim _{t \rightarrow \infty} v(t)=v^{\infty}$, the solution $x\left(t, x^{0}, v\right)$ of (4.20) converges to $x^{\infty}$ as $t \rightarrow \infty$.

Let $\xi_{0} \in(1,6)$. Then it is not difficult to show that there exists $f \in \mathcal{F}$ such that

$$
\sup _{z \neq 0} \frac{\left|f\left(z+\xi_{0}\right)-f\left(\xi_{0}\right)\right|}{|z|}=\sup _{z \neq 0} \frac{f\left(z+\xi_{0}\right)-f\left(\xi_{0}\right)}{z}>1
$$


and it is clear that condition (A) does not hold for $\xi=\xi_{0}$. We claim that, for $v^{\infty}=$ $\left(v_{1}^{\infty}, v_{2}^{\infty}\right)^{\mathrm{T}} \in \mathbb{R}^{2}$ such that

$$
\left(5 v_{1}^{\infty}+3 v_{2}^{\infty}\right) / 4=-C A^{-1} v^{\infty}=F_{0}\left(\xi_{0}\right)
$$

there does not exist $x^{\infty} \in \mathbb{R}^{2}$ such that $\lim _{t \rightarrow \infty} x\left(t, x^{0}, v\right)=x^{\infty}$ for all $x^{0} \in \mathbb{R}^{2}$ and all $v \in L^{\infty}\left(\mathbb{R}_{+}, \mathbb{R}^{2}\right)$ with $\lim _{t \rightarrow \infty} v(t)=v^{\infty}$. To this end note that, by (4.22), there exists $z_{0} \neq 0$ such that

$$
\frac{f\left(z_{0}+\xi_{0}\right)-f\left(\xi_{0}\right)}{z_{0}}>1,
$$

and thus

$$
z_{0}\left(F_{0}\left(z_{0}+\xi_{0}\right)-F_{0}\left(\xi_{0}\right)\right)<0
$$

Now (4.21) guarantees that

$$
F_{0}(z) \rightarrow \pm \infty \quad \text { as } z \rightarrow \pm \infty
$$

and hence there exists $\xi_{1} \neq \xi_{0}$ such that $F_{0}\left(\xi_{0}\right)=F_{0}\left(\xi_{1}\right)$. As a consequence, $\# F_{0}^{-1}\left(-C A^{-1} v^{\infty}\right)>1$, and so, by statement (2) of Proposition 3.1, it follows that there does not exist $x^{\infty} \in \mathbb{R}^{2}$ such that $\lim _{t \rightarrow \infty} x\left(t, x^{0}, v\right)=x^{\infty}$ for all $x^{0} \in \mathbb{R}^{2}$ and all $v \in L^{\infty}\left(\mathbb{R}_{+}, \mathbb{R}^{2}\right)$ with $\lim _{t \rightarrow \infty} v(t)=v^{\infty}$.

To illustrate the last point, we consider a specific example: fix $\xi_{0}=7 / 2 \in(1,6)$ and let $f \in \mathcal{F}$ be given by

$$
f(z):= \begin{cases}\frac{z}{2} & z \in(-\infty, 3) \\ q(z) & z \in[3,4] \\ \frac{z-4}{2}+3 & z \in(4, \infty),\end{cases}
$$

where $q(z):=-2 z^{3}+21 z^{2}-143 z / 2+81$ (Fig. 3).

It is straightforward to verify that the function $f$ belongs to $\mathcal{F}$, in particular:

$$
f(3)=3 / 2, \quad f(4)=3, \quad f^{\prime}(3)=1 / 2=f^{\prime}(4) \quad \text { and } \max _{z \in[3,4]} f^{\prime}(z)=f^{\prime}\left(\xi_{0}\right)=2 .
$$

The last identity shows that condition (4.22) holds. Moreover, $F_{0}\left(\xi_{0}\right)=\xi_{0}-f\left(\xi_{0}\right)=$ $5 / 4$, and thus, $v^{\infty}:=(2,-5 / 3)^{\mathrm{T}}$ satisfies (4.23). A straightforward argument shows that $F_{0}^{-1}(5 / 4)=\{5 / 2,7 / 2,9 / 2\}$. Calculating $x^{\infty}=-A^{-1}\left(B f\left(y^{\infty}\right)+v^{\infty}\right)$ for $y^{\infty} \in\{5 / 2,7 / 2,9 / 2\}$, we see that $\left(x^{\infty}, v^{\infty}\right)$ is an equilibrium pair for every $x^{\infty}$ of the form

$$
x^{\infty}=\left(\begin{array}{c}
13 / 12 \\
x_{2}^{\infty}
\end{array}\right), \quad \text { where } x_{2}^{\infty} \in\{1 / 3,4 / 3,7 / 3\}
$$


Fig. 3 Graph of $f$ from (4.24)

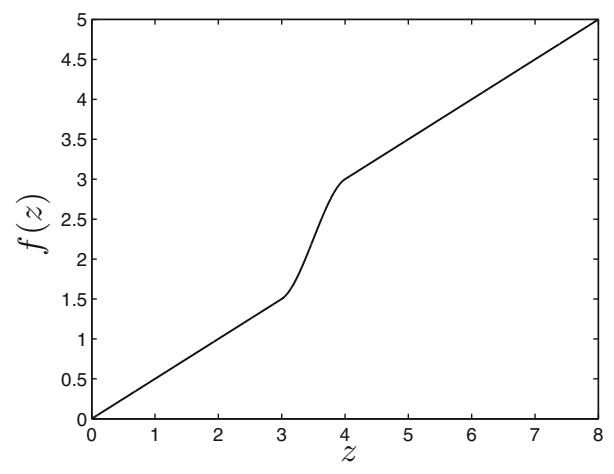

In particular, there does not exist $x^{\infty} \in \mathbb{R}^{2}$ such that $\lim _{t \rightarrow \infty} x\left(t, x^{0}, v\right)=x^{\infty}$ for all $x^{0} \in \mathbb{R}^{2}$ and all $v \in L^{\infty}\left(\mathbb{R}_{+}, \mathbb{R}^{2}\right)$ with $\lim _{t \rightarrow \infty} v(t)=v^{\infty}$.

The following corollary is a consequence of statement (1) of Theorem 4.3.

Corollary 4.7 Let $K \in \mathbb{S}_{\mathbb{R}}(A, B, C)$, set $\gamma:=1 /\left\|\mathbf{G}_{K}\right\|_{H^{\infty}}$ (where $\gamma:=\infty$ if $\left.\left\|\mathbf{G}_{K}\right\|_{H^{\infty}}=0\right)$, assume that (A) holds with $Y:=F_{K}^{-1}(\operatorname{im} C) \subseteq \operatorname{im} C$ and set $V:=$ $\left\{w \in \mathbb{R}^{n}:-C A_{K}^{-1} w \in F_{K}(Y)\right\}$. Furthermore, assume that, for every $x^{0} \in \mathbb{R}^{n}$ and every $v \in L^{\infty}\left(\mathbb{R}_{+}, \mathbb{R}^{n}\right)$ such that $\lim _{t \rightarrow \infty} v(t)=v^{\infty} \in V$, the function $C x\left(\cdot ; x^{0}, v\right)$ is bounded. Then, for every $v^{\infty} \in V$, \#F $F_{K}^{-1}\left(-C A_{K}^{-1} v^{\infty}\right)=1$ and, for every $x^{0} \in$ $\mathbb{R}^{n}$ and every $v \in L^{\infty}\left(\mathbb{R}_{+}, \mathbb{R}^{n}\right)$ such that $\lim _{t \rightarrow \infty} v(t)=v^{\infty} \in V$, we have that $x\left(t ; x^{0}, v\right) \rightarrow x^{\infty}$ as $t \rightarrow \infty$, where $x^{\infty}:=-A_{K}^{-1}\left(B\left(f\left(y^{\infty}\right)-K y^{\infty}\right)+v^{\infty}\right)$ with $y^{\infty}$ given by $\left\{y^{\infty}\right\}=F_{K}^{-1}\left(-C A_{K}^{-1} v^{\infty}\right)$.

Note that usually rank $C=p$, in which case im $C=\mathbb{R}^{p}, Y=\mathbb{R}^{p}$ and $F_{K}(Y)=$ $F_{K}\left(\mathbb{R}^{p}\right)=\operatorname{im} F_{K}$.

Proof of Corollary 4.7. Let $v^{\infty} \in V$ and set $z:=-C A_{K}^{-1} v^{\infty}$. Obviously, $z \in \operatorname{im} C$ and it follows from the definitions of the sets $Y$ and $V$ that $F_{K}^{-1}(z) \cap Y \neq \emptyset$. Consequently, by Proposition 4.1, \# $F_{K}^{-1}\left(-C A_{K}^{-1} v^{\infty}\right)=\# F_{K}^{-1}(z)=1$.

To prove the convergence property, let $x^{0} \in \mathbb{R}^{n}, v^{\infty} \in V$ and let $v \in L^{\infty}\left(\mathbb{R}_{+}, \mathbb{R}^{n}\right)$ be such that $v(t) \rightarrow v^{\infty}$ as $t \rightarrow \infty$ and write $x(t):=x\left(t ; x^{0}, v\right)$. By hypothesis, $C x$ is bounded, and so, since $x$ satisfies $\dot{x}=A_{K} x+B(f(C x)-K C x)+v$, the Hurwitz property of $A_{K}$ guarantees that $x$ is bounded. An application of statement (1) of Theorem 4.3 shows that $x(t) \rightarrow x^{\infty}$ as $t \rightarrow \infty$, completing the proof.

The next result, a corollary of statement (2) of Theorem 4.3, provides a sufficient condition for the CICS property.

Corollary 4.8 Let $K \in \mathbb{S}_{\mathbb{R}}(A, B, C)$ and set $\gamma:=1 /\left\|\mathbf{G}_{K}\right\|_{H^{\infty}}$ (where $\gamma:=\infty$ if $\left.\left\|\mathbf{G}_{K}\right\|_{H^{\infty}}=0\right)$ ). If there exists $\zeta \in \mathbb{R}^{p}$ such that (4.9) holds and $f$ satisfies

(B) $\|f(z+\xi)-f(\xi)-K z\|<\gamma\|z\|$ for all $\xi \in \operatorname{im} C$ and all $z \in \mathbb{R}^{p}, z \neq 0$, then (1.1) has the CICS property. 
Proof The map $F_{K}$ is surjective, as follows from hypothesis (B), (4.9) and statement (3) of Proposition 4.1. Hence, by (3.1),

$$
F_{K}^{-1}\left(-C A_{K}^{-1} v^{\infty}\right) \cap \operatorname{im} C \neq \emptyset \quad \forall v^{\infty} \in \mathbb{R}^{n}
$$

Invoking statement (2) of Theorem 4.3 (with $Y=\operatorname{im} C$ ) shows that the Lur'e system (1.1) has the CICS property.

As an illustration of Corollary 4.7, consider the system (4.18) with $f$ given by (4.19) and $K=0$, see part (a) of Example 4.5. In this case, $\gamma=1, Y=\mathbb{R}$ and $V=$ $F_{0}(\mathbb{R})=(-1,1)$. As has been shown in part (a) of Example (4.5), assumption (A) holds with $Y=\mathbb{R}$ and $C x\left(\cdot ; x^{0}, v\right)=x\left(\cdot ; x^{0}, v\right)$ is bounded for all $x^{0} \in \mathbb{R}$ and all convergent $v \in L^{\infty}\left(\mathbb{R}_{+}, \mathbb{R}^{n}\right)$ with limit in $(-1,1)$. Consequently, all assumptions of Corollary 4.7 are satisfied and so, for all $x^{0} \in \mathbb{R}$ and all $v \in L^{\infty}\left(\mathbb{R}_{+}, \mathbb{R}^{n}\right)$ such that $\lim _{t \rightarrow \infty} v(t)=v^{\infty}$, we have that $\lim _{t \rightarrow \infty} x\left(t ; x^{0}, v\right)=x^{\infty}$, where $x^{\infty}$ is given by $\left\{x^{\infty}\right\}=F_{0}^{-1}\left(v^{\infty}\right)$. Note that the system does not have the CICS property, since the input $v(t) \equiv 1+\varepsilon, \varepsilon>0$, generates a divergent state trajectory. Moreover, note that Corollary 4.8 does not apply: whilst assumption (B) is satisfied, there does not exist $\zeta \in \mathbb{R}$ such that (4.9) holds.

We give a sufficient condition for (B) to hold. The proof is routine and is, therefore, left to the reader.

Lemma 4.9 Assume that $f: \mathbb{R}^{p} \rightarrow \mathbb{R}^{m}$ is continuously differentiable, with derivative denoted by $D f$. Let $\Delta \subseteq \mathbb{R}^{p}$ be a set which does not have any accumulation points. If

$$
\|(D f)(z)-K\|<\gamma \quad \forall z \in \mathbb{R}^{p} \backslash \Delta
$$

then condition (B) holds.

In the following, we shall derive a number of further corollaries which will provide "interpretations" of Corollary 4.8 in terms of the complex Aizerman conjecture, smallgain theorems and circle criteria, respectively.

The next result is reminiscent of the complexified Aizerman conjecture $[17,18,36]$.

Corollary 4.10 Let $K \in \mathbb{R}^{m \times p}, r>0$ and assume that $\mathbb{B}_{\mathbb{C}}(K, r) \subseteq \mathbb{S}_{\mathbb{C}}(A, B, C)$. If

$$
\|f(z+\xi)-f(\xi)-K z\|<r\|z\| \quad \forall \xi \in \operatorname{im} C, \forall z \in \mathbb{R}^{p}, z \neq 0
$$

and there exists $\zeta \in \mathbb{R}^{p}$ such that

$$
r\|z\|-\|f(z+\zeta)-f(\zeta)-K z\| \rightarrow \infty \text { as }\|z\| \rightarrow \infty
$$

then (1.1) has the CICS property.

Corollary 4.10 says, roughly speaking, that linear stability (namely $\mathbb{B}_{\mathbb{C}}(K, r) \subseteq$ $\left.\mathbb{S}_{\mathbb{C}}(A, B, C)\right)$ implies CICS for all nonlinearities $f$ satisfying the "incremental" ball condition (4.25) and the divergence property (4.26). 
Proof of Corollary 4.10. By hypothesis $\mathbb{B}_{\mathbb{C}}(K, r) \subseteq \mathbb{S}_{\mathbb{C}}(A, B, C)$ and so, $A_{K}=$ $A+B K C$ is Hurwitz and $\mathbb{B}_{\mathbb{C}}(0, r) \subseteq \mathbb{S}_{\mathbb{C}}\left(A_{K}, B, C\right)$. Thus, appealing to elementary stability radius theory $[16,18]$ (see also the proof of Theorem 2.1 in the Appendix), we have that $r \leq 1 /\|\mathbf{G}\|_{H^{\infty}}$. The claim follows now from Corollary 4.8.

Consider the following incremental small-gain condition:

$\left(\mathbf{B}^{\prime}\right)$ For every $\xi \in \operatorname{im} C$, there exists $\alpha_{\xi} \in \mathcal{K}_{\infty}$ such that

$$
\left\|\mathbf{G}_{K}\right\|_{H^{\infty}} \frac{\|f(z+\xi)-f(\xi)-K z\|}{\|z\|} \leq 1-\frac{\alpha_{\xi}(\|z\|)}{\|z\|} \quad \forall z \in \mathbb{R}^{p}, z \neq 0,
$$

We are now in the position to state a "nonlinear" small-gain criterion for the CICS property.

Corollary 4.11 Let $K \in \mathbb{S}_{\mathbb{R}}(A, B, C)$. If $f$ satisfies $\left(\mathrm{B}^{\prime}\right)$, then (1.1) has the CICS property.

Proof It is clear that if $\left(\mathrm{B}^{\prime}\right)$ is satisfied, then (B) and (4.9) hold. Thus, the claim follows from Corollary 4.8 .

Note that $\left(\mathrm{B}^{\prime}\right)$ is not a small-gain condition in the sense of classical input-output theory of feedback systems (as presented, for example, in $[11,13,14,23,45]$ ): whilst, for every fixed $\xi \in \operatorname{im} C$, the right-hand side of (4.27) is smaller than 1 for all $z \neq 0$, it is in general not uniformly bounded away from 1. Indeed, it is possible that, for fixed $\xi$, the right-hand side of (4.27) is converging to 1 as $\|z\| \rightarrow 0$ or $\|z\| \rightarrow \infty$. Therefore, rather than comparing Corollary 4.11 with classical small-gain theorems $[11,13,14,23,45]$, it is more appropriate to view it in the context of "modern" nonlinear ISS small-gain results, see for example [10,21,36,43].

If $\operatorname{im} C=\mathbb{R}^{p}$, then condition (B) implies that $f_{K}: \mathbb{R}^{p} \rightarrow \mathbb{R}^{m}, z \mapsto f(z)-K z$ is globally Lipschitz and $\gamma$ is a Lipschitz constant for $f_{K}$. If the map $f_{K}$ is globally Lipschitz and has a Lipschitz constant $\lambda<\gamma$, then

$$
\left\|\mathbf{G}_{K}\right\|_{H^{\infty}} \frac{\left\|f_{K}(z+\xi)-f_{K}(\xi)\right\|}{\|z\|} \leq \frac{\lambda}{\gamma}<1 \quad \forall z, \xi \in \mathbb{R}^{p}, z \neq 0 .
$$

This inequality, a (incremental) small-gain condition in the sense of classical inputoutput theory, is sufficient for $\left(\mathrm{B}^{\prime}\right)$ to hold. Consequently, (4.28) is a sufficient condition for the CICS property.

In the following example, we present a simple nonlinearity $f$ such that $f$ satisfies condition (B), $f_{K}$ has minimal Lipschitz constant equal to $\gamma$ and (4.9) holds.

Example 4.12 Let

$$
A:=\left(\begin{array}{ccc}
-2 & -1 & 0 \\
1 & -1 & -1 \\
-1 & 0 & 0
\end{array}\right), \quad B:=\left(\begin{array}{l}
0 \\
0 \\
1
\end{array}\right), \quad C:=(1,0,0)
$$


The characteristic polynomial of $A$ is $\operatorname{det}(s I-A)=(s+1)^{3}$. Hence, $A$ is Hurwitz and so may choose $K=0$, leading to

$$
\mathbf{G}_{K}(s)=\mathbf{G}_{0}(s)=\mathbf{G}(s)=\frac{1}{(s+1)^{3}} .
$$

A routine argument shows that

$$
\|\mathbf{G}\|_{H^{\infty}}=\mathbf{G}(0)=1,
$$

and thus $\gamma=1 /\|\mathbf{G}\|_{H^{\infty}}=1$. In the following, we consider the Lur'e system

$$
\dot{x}=A x+B f(C x)+v, \quad \text { where } f(z)=\operatorname{sign}(z) \ln (1+|z|) .
$$

The function $f$ is continuously differentiable and

$$
f^{\prime}(0)=1 \quad \text { and } \quad 0<f^{\prime}(z)<1 \quad \forall z \neq 0 .
$$

It follows from Lemma 4.9 that condition (B) is satisfied. Moreover, trivially, $|z|-$ $|f(z)| \rightarrow \infty$ as $|z| \rightarrow \infty$, and so, Corollary 4.8 guarantees that (4.29) has the CICS property. Finally, note that $f$ is globally Lipschitz with minimal Lipschitz constant equal to $\gamma=1$.

Remark 4.13 If the assumptions of Corollary 4.8 hold, then, by Proposition 4.1, the map $\hat{F}_{K}: \operatorname{im} C \rightarrow \operatorname{im} C$ restricting $F_{K}$ to im $C$ is bijective and the ISSS gain of (1.1) can be written as

$$
\Gamma_{\text {is }}(z)=-A_{K}^{-1}\left(B\left(f_{K} \circ \hat{F}_{K}^{-1}\right)\left(-C A_{K}^{-1} z\right)+z\right) \quad \forall z \in \mathbb{R}^{n},
$$

where $f_{K}(z):=f(z)-K z$. Similarly, the IOSS gain of (1.1) can expressed as

$$
\Gamma_{\text {io }}(z)=\hat{F}_{K}^{-1}\left(-C A_{K}^{-1} z\right) \quad \forall z \in \mathbb{R}^{n} .
$$

Note that if $A$ is Hurwitz, $f=0$ and $K=0$, then (1.1) "collapses" to the linear system

$$
\dot{x}=A x+v, \quad y=C x,
$$

which has transfer function $\mathbf{H}(s)=C(s I-A)^{-1}$. In this case, $F_{K}(z)=F_{0}(z)=z$ for all $z \in \mathbb{R}^{n}$ and $\Gamma_{\text {io }}(z)=-C A^{-1} z=\mathbf{H}(0) z$, that is, the familiar linear steady-state gain is recovered.

Statement (1) of the next proposition demonstrates that, under the assumptions of Corollary 4.8, the maps $\Gamma_{\text {is }}$ and $\Gamma_{\text {io }}$ (the steady-state gains) are continuous.

Proposition 4.14 Let $K \in \mathbb{S}_{\mathbb{R}}(A, B, C)$ and set $\gamma:=1 /\left\|\mathbf{G}_{K}\right\|_{H^{\infty}}($ where $\gamma:=\infty$ if $\left.\left\|\mathbf{G}_{K}\right\|_{H^{\infty}}=0\right)$. 
1. If the assumptions of Corollary 4.8 hold, then the steady-state gains $\Gamma_{\text {is }}$ and $\Gamma_{\text {io }}$ are continuous.

2. If the map $\mathbb{R}^{p} \rightarrow \mathbb{R}^{m}, z \mapsto f(z)-K z$ is globally Lipschitz with Lipschitz constant $\lambda<\gamma$, then $\Gamma_{\text {is }}$ and $\Gamma_{\text {io }}$ are globally Lipschitz.

Proof To prove statement (1), we note that, in light of Remark 4.13, it is sufficient to show that $\hat{F}_{K}^{-1}: \operatorname{im} C \rightarrow \operatorname{im} C$ is continuous. To this end, let $w \in \operatorname{im} C$ be fixed, but arbitrary, set $\xi:=\hat{F}_{K}^{-1}(w) \in \operatorname{im} C$ and define $\tilde{f}: \mathbb{R}^{p} \rightarrow \mathbb{R}^{p}$ by $\tilde{f}(z):=$ $f(z+\xi)-f(\xi)$ for all $z \in \mathbb{R}^{p}$. Invoking the divergence assumption (4.9) and statement (1) of Lemma 4.2 shows that

$$
\gamma\|z\|-\|\tilde{f}(z)-K z\| \rightarrow \infty \text { as }\|z\| \rightarrow \infty
$$

and so by statement (2) of Lemma 4.2, there exists $\alpha \in \mathcal{K}_{\infty}$ such that

$$
\frac{1}{\gamma}\|\tilde{f}(z)-K z\| \leq\|z\|-\alpha(\|z\|) \quad \forall z \in \mathbb{R}^{p}
$$

We now use (4.32) to estimate

$$
\begin{aligned}
\left\|\hat{F}_{K}(z)-\hat{F}_{K}(\xi)\right\| & =\left\|z-\mathbf{G}_{K}(0)(f(z)-K z)-\left(\xi-\mathbf{G}_{K}(0)(f(\xi)-K \xi)\right)\right\| \\
& =\|z-\xi\|-\frac{1}{\gamma}\|\tilde{f}(z-\xi)-K(z-\xi)\| \\
& \geq \alpha(\|z-\xi\|) \quad \forall z \in \operatorname{im} C .
\end{aligned}
$$

Thus,

$$
\|z-\xi\| \leq \alpha^{-1}\left(\left\|\hat{F}_{K}(z)-\hat{F}_{K}(\xi)\right\|\right) \quad \forall z \in \operatorname{im} C,
$$

where $\alpha^{-1} \in \mathcal{K}_{\infty}$, as $\alpha \in \mathcal{K}_{\infty}$. Invoking the invertibility of $\hat{F}_{K}$ and the definition of $\xi$, we see that

$$
\left\|\hat{F}_{K}^{-1}(z)-\hat{F}_{K}^{-1}(w)\right\| \leq \alpha^{-1}(\|z-w\|) \quad \forall z \in \operatorname{im} C .
$$

In particular, we obtain that $\hat{F}_{K}^{-1}$ is continuous at $w$. As $w \in \operatorname{im} C$ was arbitrary, continuity of $\hat{F}_{K}^{-1}$ follows.

The proof of statement (2) is similar to that of statement (1). Indeed, we have that

$$
\begin{aligned}
\left\|\hat{F}_{K}(z)-\hat{F}_{K}(\xi)\right\| & \geq\|z-\xi\|-\frac{1}{\gamma}\|f(z)-K z-(f(\xi)-K \xi)\| \\
& \geq\left(1-\frac{\lambda}{\gamma}\right)\|z-\xi\| \quad \forall z, \xi \in \operatorname{im} C,
\end{aligned}
$$

which implies that

$$
\left\|\hat{F}_{K}^{-1}(z)-\hat{F}_{K}^{-1}(\xi)\right\| \leq \frac{\gamma}{\gamma-\lambda}\|z-\xi\| \quad \forall z, \xi \in \operatorname{im} C,
$$


showing that $\hat{F}_{K}^{-1}$ is globally Lipschitz. Consequently, invoking formulas (4.30) and (4.31), the steady-state gains $\Gamma_{\text {is }}$ and $\Gamma_{\text {io }}$ inherit the global Lipschitz property from that of their constituents.

Next we present, in form of two corollaries, sufficient conditions for the CICS property which are reminiscent of the well-known circle criterion (see, for example, $[14,23,36,45])$.

Corollary 4.15 Let $K_{1}, K_{2} \in \mathbb{R}^{m \times p}$. Assume that $(A, B, C)$ is stabilizable and detectable, $\left(I-K_{2} \mathbf{G}\right)\left(I-K_{1} \mathbf{G}\right)^{-1}$ is positive real,

$\left\langle f(z+\xi)-f(\xi)-K_{1} z, f(z+\xi)-f(\xi)-K_{2} z\right\rangle<0 \quad \forall \xi \in \operatorname{im} C, \forall z \in \mathbb{R}^{p}, z \neq 0$,

and there exist $\zeta \in \mathbb{R}^{p}$ and $\alpha \in \mathcal{K}_{\infty}$ such that

$$
\left\langle f(z+\zeta)-f(\zeta)-K_{1} z, f(z+\zeta)-f(\zeta)-K_{2} z\right\rangle \leq-\alpha(\|z\|)\|z\| \quad \forall z \in \mathbb{R}^{p}
$$

Then the Lur'e system (1.1) has the CICS property.

Proof We shall rewrite the Lur'e system in a form which will allow the application of Corollary 4.8. For $\xi \in \mathbb{R}^{p}$, define $f_{\xi}: \mathbb{R}^{p} \rightarrow \mathbb{R}^{m}$ by

$$
f_{\xi}(z)=f(z+\xi)-f(\xi) \quad \forall z \in \mathbb{R}^{p}
$$

Setting

$$
L:=\frac{1}{2}\left(K_{1}-K_{2}\right) \text { and } M:=\frac{1}{2}\left(K_{1}+K_{2}\right)
$$

we have that

$$
\begin{aligned}
\left\langle f_{\xi}(z)-K_{1} z, f_{\xi}(z)-K_{2} z\right\rangle & =\left\langle f_{\xi}(z)-(M+L) z, f_{\xi}(z)-(M-L) z\right\rangle \\
& =\left\|f_{\xi}(z)-M z\right\|^{2}-\|L z\|^{2} \quad \forall z \in \mathbb{R}^{p}
\end{aligned}
$$

Note that in conjunction with (4.33) (or, alternatively, (4.34) could be invoked) this implies ker $L=\{0\}$. Thus $L^{*} L$ is invertible and $L^{\sharp}:=\left(L^{*} L\right)^{-1} L^{*} \in \mathbb{R}^{p \times m}$ is a left inverse of $L$. Define the nonlinearity $g: \mathbb{R}^{m} \rightarrow \mathbb{R}^{m}$ by $g(z):=f\left(L^{\sharp} z\right)-K_{1} L^{\sharp} z$ for all $z \in \mathbb{R}^{m}$ and consider the Lur'e system

$$
\dot{x}=A_{K_{1}} x+B g(L C x)+v,
$$

where $A_{K_{1}}:=A+B K_{1} C$. The linear state space system $\left(A_{K_{1}}, B, L C\right)$ has transfer function

$$
\mathbf{H}(s)=L C\left(s I-A_{K_{1}}\right)^{-1} B=L \mathbf{G}_{K_{1}}(s), \quad \text { where } \mathbf{G}_{K_{1}}=\mathbf{G}\left(I-K_{1} \mathbf{G}\right)^{-1} .
$$


It is obvious that $x$ is solves the original Lur'e system $\dot{x}=A x+B f(C x)+v$ if, and only if, $x$ solves (4.36). Therefore, it is sufficient to show that (4.36) has the CICS property. To this end, set $K:=-L L^{\sharp}$. Using, mutatis mutandis, arguments from [36, proof of Corollary 3.10], it follows that $K \in \mathbb{S}_{\mathbb{R}}\left(A_{K_{1}}, B, L C\right)$,

$$
\gamma:=1 /\left\|\mathbf{H}_{K}\right\|_{H^{\infty}} \geq 1, \quad \text { where } \mathbf{H}_{K}:=\mathbf{H}(I-K \mathbf{H})^{-1},
$$

there exists $\beta \in \mathcal{K}_{\infty}$ such that

$$
\|g(z+L \zeta)-g(L \zeta)-K z\| \leq\|z\|-\beta(\|z\|) \leq \gamma\|z\|-\beta(\|z\|) \quad \forall z \in \mathbb{R}^{m},
$$

and

$$
\|g(z+\eta)-g(\eta)-K z\|<\|z\| \leq \gamma\|z\| \quad \forall \eta \in \operatorname{im}(L C), \forall z \in \mathbb{R}^{m}, z \neq 0 .
$$

Consequently, the assumptions of Corollary 4.8 are satisfied in the context of the Lur'e system (4.36) and therefore, (4.36) has the CICS property, completing the proof.

Recall that a rational square matrix $\mathbf{H}$ is said to be strictly positive real if there exists $\varepsilon>0$ such the rational matrix function $s \mapsto \mathbf{H}(s-\varepsilon)$ is positive real.

Corollary 4.16 Let $K_{1}, K_{2} \in \mathbb{R}^{m \times p}$. Assume that $\operatorname{ker}\left(K_{1}-K_{2}\right)=\{0\},(A, B, C)$ is stabilisable and detectable, $\left(I-K_{2} \mathbf{G}\right)\left(I-K_{1} \mathbf{G}\right)^{-1}$ is strictly positive real and

$$
\left\langle f(z+\xi)-f(\xi)-K_{1} z, f(z+\xi)-f(\xi)-K_{2} z\right\rangle \leq 0 \quad \forall \xi \in \operatorname{im} C, \forall z \in \mathbb{R}^{p}
$$

Then the Lur'e system (1.1) has the CICS property.

Proof Set $M:=K_{2}-K_{1}$, let $\xi \in \operatorname{im} C$ and define $f_{\xi}: \mathbb{R}^{p} \rightarrow \mathbb{R}^{m}$ by (4.35). Then, mutatis mutandis, arguments from [36, proof of Corollary 3.13] can be invoked to show that there exists $k>0$ and $\mu>0$ such that, for all $\kappa \in(0, k)$, the rational matrix function

$$
\left(I-\left(K_{2}+\kappa M\right) \mathbf{G}\right)\left(I-\left(K_{1}-\kappa M\right) \mathbf{G}\right)^{-1}
$$

is positive real and

$$
\left\langle f_{\xi}(z)-\left(K_{1}-\kappa M\right) z, f_{\xi}(z)-\left(K_{2}+\kappa M\right) z\right\rangle \leq-\mu \kappa(\kappa+1)\|z\|^{2} \quad \forall z \in \mathbb{R}^{p} .
$$

It follows that the conditions of Corollary 4.15 hold (with $\alpha(s)=\mu \kappa(\kappa+1) s$ and $K_{1}$ and $K_{2}$ replaced by $K_{1}-\kappa M$ and $K_{2}+\kappa M$, respectively). Hence, (1.1) has the CICS property.

Note that the assumptions in Corollary 4.16 are essentially identical to those in the "classical" circle criterion which guarantees global asymptotic stability (see [13, Theorem 5.1], [14, Corollary 5.8] and [23, Theorem 7.1], the only difference being 
that (4.37) is the incremental version of the standard sector condition in the circle criterion.

We further note that Corollary 4.16 is reminiscent of the main result in [35] which provides a description of the steady-state error of single-input single-output Lur'e systems of the form (1.2) in response to a class of polynomial inputs (including unbounded signals such as ramps) under the assumption that the conditions of the SISO circle criterion are met. Whilst the CICS property is not mentioned in [35], part (1) of [35, Theorem] can be interpreted in CICS terms.

We emphasize that Corollaries 4.15 and 4.16 are not equivalent. Indeed, the latter is more conservative than the former as is illustrated by the following simple example.

Example 4.17 Consider the one-dimensional Lur'e system

$$
\dot{x}(t)=f(x(t))+v(t) \quad x(0)=x^{0} \quad t \geq 0,
$$

with $f: \mathbb{R} \rightarrow \mathbb{R}$ given by $f(z)=-\operatorname{sign}(z) \ln (1+|z|)$. The function $f$ is continuously differentiable, $f^{\prime}(0)=-1$ and $-1<f^{\prime}(z)<0$ for all $z \neq 0$. Obviously, (4.38) is of the form (1.1) with $(A, B, C)=(0,1,1)$, and so $\mathbf{G}(s)=1 / s$. Let $K_{1}<K_{2}$ and note that

$$
\frac{1-K_{2} \mathbf{G}(s)}{1-K_{1} \mathbf{G}(s)}=\frac{s-K_{2}}{s-K_{1}}
$$

is (strictly) positive real if, and only if, $K_{2} \leq 0\left(K_{2}<0\right)$.

Now if $K_{1}<K_{2}<0$, then, for every $\xi \in \mathbb{R}$,

$$
\left(f(z+\xi)-f(\xi)-K_{1} z\right)\left(f(z+\xi)-f(\xi)-K_{2} z\right)>0, \quad|z| \text { sufficiently large }
$$

and we conclude that Corollary 4.16 does not apply.

However, choosing $K_{1}<-1$ and $K_{2}=0$, it is not difficult to show that the conditions of Corollary 4.15 are satisfied. Indeed, for $K_{1}<-1$ and $K_{2}=0$, the rational function in (4.39) is positive real and, by the mean-value theorem for differentiation,

$$
\left(f(z+\xi)-f(\xi)-K_{1} z\right)(f(z+\xi)-f(\xi))<0 \quad \forall \xi, z \in \mathbb{R}, z \neq 0
$$

Furthermore, it is clear that

$$
\operatorname{sign}(z)\left(f(z) / z-K_{1}\right) f(z) \rightarrow-\infty \text { as }|z| \rightarrow \infty
$$

which, together with (4.40), shows that there exists $\alpha \in \mathcal{K}_{\infty}$ such that

$$
\left(f(z)-K_{1} z\right) f(z) \leq-\alpha(|z|)|z| \quad \forall z \in \mathbb{R} .
$$

We have now established that the assumptions of Corollary 4.15 hold (with $K_{1}<$ $-1, K_{2}=0$ and $\zeta=0$ ) and consequently, system (4.38) has the CICS property. 


\section{The CICS property for another class of Lur'e systems}

In this short section, we consider forced Lur'e systems of the form

$$
\left.\begin{array}{l}
\dot{x}(t)=A x(t)+B f(C x(t)-v(t)), \quad x(0)=x^{0} \in \mathbb{R}^{n} \\
y(t)=C x(t)
\end{array}\right\} \quad t \geq 0,
$$

where, as in Sects. 2-4, $A \in \mathbb{R}^{n \times n}, B \in \mathbb{R}^{n \times m}, C \in \mathbb{R}^{p \times n}, f: \mathbb{R}^{p} \rightarrow \mathbb{R}^{m}, y$ denotes the output and $v \in L_{\mathrm{loc}}^{\infty}\left(\mathbb{R}_{+}, \mathbb{R}^{p}\right)$ is the control (forcing, input) function. In the uncontrolled case $(v=0)$, the Lur'e systems (1.1) and (5.1) are identical. As has been pointed out in Sect. 1, the Lur'e system (5.1) can be thought of as a closed-loop system obtained by applying the linear feedback $w=y-v$ to the system $\dot{x}=A x+B f(w)$ (a linear system with input nonlinearity), see Fig. 2.

Let $\hat{x}\left(\cdot ; x^{0}, v\right)$ denote the unique maximally defined forward solution of the initialvalue problem (5.1). The CICS property can be defined as before: Lur'e system (5.1) is said to have the CICS property if, for every $v^{\infty} \in \mathbb{R}^{p}$, there exists $x^{\infty} \in \mathbb{R}^{n}$ such that $\lim _{t \rightarrow \infty} \hat{x}\left(t ; x^{0}, v\right)=x^{\infty}$ for all $x^{0} \in \mathbb{R}^{n}$ and all $v \in L^{\infty}\left(\mathbb{R}_{+}, \mathbb{R}^{p}\right)$ with $\lim _{t \rightarrow \infty} v(t)=v^{\infty}$.

The following corollary provides a sufficient condition for (5.1) to have the CICS property.

Proposition 5.1 Let $K \in \mathbb{S}_{\mathbb{R}}(A, B, C)$, assume that $\left\|\mathbf{G}_{K}\right\|_{H^{\infty}}>0$ and set $\gamma:=$ $1 /\left\|\mathbf{G}_{K}\right\|_{H^{\infty}}$. Furthermore, assume that there exists $\zeta \in \mathbb{R}^{p}$ such that (4.9) holds and $f$ satisfies

(C) $\|f(z+\xi)-f(\xi)-K z\|<\gamma\|z\|$ for all $\xi, z \in \mathbb{R}^{p}, z \neq 0$.

Then the map $F_{K}$ is bijective and, for all $v^{\infty} \in \mathbb{R}^{p}$, all $x^{0} \in \mathbb{R}^{n}$ and all $v \in$ $L^{\infty}\left(\mathbb{R}_{+}, \mathbb{R}^{p}\right)$ with $\lim _{t \rightarrow \infty} v(t)=v^{\infty}$,

$$
\lim _{t \rightarrow \infty} \hat{x}\left(t ; x^{0}, v\right)=x^{\infty}:=-A_{K}^{-1} B\left(f\left(y^{\infty}-v^{\infty}\right)-K y^{\infty}\right),
$$

where $y^{\infty} \in \mathbb{R}^{p}$ is given by $y^{\infty}:=F_{K}^{-1}\left(-\left(I+\mathbf{G}_{K}(0) K\right) v^{\infty}\right)+v^{\infty}$ and satisfies $y^{\infty}=C x^{\infty}$. In particular, the Lur'e system (5.1) has the CICS property.

Remark 5.2 Note that under the assumptions of Proposition 5.1, it is natural to define the IOSS gain of (5.1) to be the map $v^{\infty} \mapsto F_{K}^{-1}\left(-\left(I+\mathbf{G}_{K}(0) K\right) v^{\infty}\right)+v^{\infty}$. Proposition 5.1 allows us to extend a classical result on integral control to Lur'e systems of the form (5.1). To this end, assume that the assumptions of Proposition 5.1 are satisfied, $f(0)=0$ and the linear system $(A, B, C)$ contains an integrator, that is, $\mathbf{G}$ has a Laurent expansion of the form

$$
\mathbf{G}(s)=\sum_{j=-1}^{\infty} G_{j} s^{j} \text { for all sufficiently small }|s|, s \neq 0,
$$

where $G_{j} \in \mathbb{R}^{p \times m}$ and $G_{-1} \neq 0$. If $G_{-1} K$ is invertible, then $\mathbf{G}_{K}(0) K=-I$ and so, $y^{\infty}=F_{K}^{-1}(0)+v^{\infty}=v^{\infty}$ (where we have used that $f(0)=0$ ), showing that every 
input $v$ with limit $v^{\infty}$ produces an output $y$ converging also to $v^{\infty}$, or equivalently, the IOSS gain of (5.1) is equal to the identity.

Proof of Proposition 5.1. It follows from statement (3) of Proposition 4.1 that $F_{K}$ is surjective. Injectivity of $F_{K}$ can be shown by an argument similar to that used in the proof of statement (1) of Proposition 4.1.

To prove the convergence property, let $x^{0} \in \mathbb{R}^{n}, v^{\infty} \in \mathbb{R}^{p}$ and $v \in L^{\infty}\left(\mathbb{R}_{+}, \mathbb{R}^{p}\right)$ such that $v(t) \rightarrow v^{\infty}$ as $t \rightarrow \infty$. Setting $\tilde{x}(t):=\hat{x}\left(t ; x^{0}, v\right)-x^{\infty}, \tilde{v}(t):=v(t)-v^{\infty}$ and $\tilde{f}(z):=f\left(z+y^{\infty}-v^{\infty}\right)-f\left(y^{\infty}-v^{\infty}\right)$, a routine calculation shows that $\tilde{x}$ satisfies

$$
\dot{\tilde{x}}(t)=A \tilde{x}(t)+B \tilde{f}(C \tilde{x}(t)-\tilde{v}(t)) \text { for almost all } t \geq 0
$$

Consequently, writing $w:=B[f(C \tilde{x}-\tilde{v})-f(C \tilde{x})]$, it follows that

$$
\dot{\tilde{x}}(t)=A \tilde{x}(t)+B \tilde{f}(C \tilde{x}(t))+w(t) \text { for almost all } t \geq 0,
$$

and we note that (5.2) is a forced Lur'e system of the form (1.1). Note that the hypotheses on $f$ combined with Lemma 4.2 guarantee that there exists $\alpha \in \mathcal{K}_{\infty}$ such that $\|\tilde{f}(z)-K z\| \leq \gamma\|z\|-\alpha(\|z\|)$ for all $z \in \mathbb{R}^{p}$. Consequently, by Theorem 2.1, the equilibrium pair $(0,0)$ of $(5.2)$ is ISS. Moreover, hypothesis $(\mathrm{C})$ implies that

$$
\|w(t)\| \leq\|B\|(\gamma+\|K\|)\|\tilde{v}(t)\| \quad \forall t \geq 0
$$

showing that $w(t) \rightarrow 0$ as $t \rightarrow \infty$ (note that $\gamma<\infty$ by hypothesis). An application of Proposition 2.2 now shows that $\tilde{x}(t) \rightarrow 0$ as $t \rightarrow \infty$ and thus, $\hat{x}\left(t ; x^{0}, v\right) \rightarrow x^{\infty}$ as $t \rightarrow \infty$.

It remains to show that $y^{\infty}=C x^{\infty}$. To see this, note that $C x^{\infty}=\mathbf{G}_{K}(0)\left(f\left(y^{\infty}-\right.\right.$ $\left.\left.v^{\infty}\right)-K y^{\infty}\right)$. Hence,

$$
\begin{aligned}
y^{\infty}-C x^{\infty}= & y^{\infty}-v^{\infty}-\mathbf{G}_{K}(0)\left(f\left(y^{\infty}-v^{\infty}\right)-K\left(y^{\infty}-v^{\infty}\right)\right) \\
& +\left(I+\mathbf{G}_{K}(0) K\right) v^{\infty},
\end{aligned}
$$

and so,

$$
y^{\infty}-C x^{\infty}=F_{K}\left(y^{\infty}-v^{\infty}\right)+\left(I+\mathbf{G}_{K}(0) K\right) v^{\infty} .
$$

But $F_{K}\left(y^{\infty}-v^{\infty}\right)=-\left(I+\mathbf{G}_{K}(0) K\right) v^{\infty}$, implying that $y^{\infty}=C x^{\infty}$.

\section{CICS properties for non-negative Lur'e systems}

In this section, we study non-negative Lur'e systems, which, as has already been indicated in Sect. 1, arise naturally in a variety of applied contexts, such as population dynamics and chemical reaction models, cf. $[6,36,37,44]$. We will restrict attention 
to models with scalar feedback $f$ ( $m=p=1$ and $f$ is a scalar function), that is, we consider forced Lur'e systems of the form

$$
\left.\begin{array}{l}
\dot{x}(t)=A x(t)+b f\left(c^{\mathrm{T}} x(t)\right)+v(t), \quad x(0)=x^{0} \in \mathbb{R}_{+}^{n} \\
y(t)=c^{\mathrm{T}} x(t)
\end{array}\right\} t \geq 0,
$$

so that, in particular, the linear system $\left(A, b, c^{\mathrm{T}}\right)$ is a single-input, single-output (SISO) system. We assume that the following positivity conditions hold:

(P1) $A \in \mathbb{R}^{n \times n}$ is Metzler and $b, c \in \mathbb{R}_{+}^{n}, b, c>0$,

(P2) $f: \mathbb{R}_{+} \rightarrow \mathbb{R}_{+}$is locally Lipschitz.

Furthermore, we only consider non-negative control (forcing, input) functions, that is, $v \in L_{\text {loc }}^{\infty}\left(\mathbb{R}_{+}, \mathbb{R}_{+}^{n}\right)$.

As before, we denote the unique maximally defined forward solution of the initialvalue problem $(6.1)$ by $x\left(\cdot ; x^{0}, v\right)$. It is well known that if (P1) and (P2) hold, then for all non-negative initial states $x^{0} \in \mathbb{R}_{+}^{n}$ and $v \in L_{\text {loc }}^{\infty}\left(\mathbb{R}_{+}, \mathbb{R}_{+}^{n}\right)$, the solution $x\left(t ; x^{0}, v\right)$ remains in the non-negative orthant $\mathbb{R}_{+}^{n}$ for all $t \in[0, \omega)$, where $[0, \omega), 0<\omega \leq \infty$ denotes the maximal interval of existence. If $\omega<\infty$, then $\left\|x\left(t ; x^{0}, v\right)\right\| \rightarrow \infty$ as $t \rightarrow \omega$. If (P1) and (P2) hold, then we will refer to (6.1) as a non-negative Lur'e system.

For later purposes, we introduce a further positivity assumption on the linear system $\left(A, b, c^{\mathrm{T}}\right)$.

(P3) The matrix $A+b c^{\mathrm{T}}$ is irreducible.

Note that $A+b c^{\mathrm{T}}$ is irreducible if, and only, if $A+k b c^{\mathrm{T}}$ is irreducible for every $k>0$.

Let $s \mapsto \mathbf{G}(s)=c^{\mathrm{T}}(s I-A)^{-1} b$ denote the transfer function of the linear SISO system $\left(A, b, c^{\mathrm{T}}\right)$. A proof of the following result can be found in [6].

Proposition 6.1 If A is Hurwitz and (P1) holds, then

$$
\|\mathbf{G}\|_{H^{\infty}}=\mathbf{G}(0) \geq 0 .
$$

Under the additional assumption that $(\mathrm{P} 3)$ is satisfied we have

$$
\|\mathbf{G}\|_{H^{\infty}}=\mathbf{G}(0)>0 .
$$

It follows from Proposition 6.1 that if $A$ is Hurwitz, (P1) holds and $(A, b)$ is controllable or $\left(c^{\mathrm{T}}, A\right)$ is observable, then $\mathbf{G}(0)>0$.

Theorem 6.2 Let $Y \subseteq \mathbb{R}_{+}$be nonempty and assume that (P1) and (P2) hold and $A$ is Hurwitz. Set $\gamma:=1 / \mathbf{G}(0)$ (where $\gamma:=\infty$ if $\mathbf{G}(0)=0$ ) and assume further that

$$
\left|\frac{f(z)-f(\xi)}{z-\xi}\right|<\gamma \quad \forall(\xi, z) \in Y \times \mathbb{R}_{+} \text {such that } z \neq \xi,
$$

and

$$
\gamma z-f(z) \rightarrow \infty \text { as } z \rightarrow \infty
$$


Then the following statements hold.

1. The map

$$
F: \mathbb{R}_{+} \rightarrow \mathbb{R}, \quad z \mapsto z-\mathbf{G}(0) f(z)
$$

has the following properties: $\mathbb{R}_{+} \subseteq F\left(\mathbb{R}_{+}\right)$and $\# F^{-1}(z)=1$ for every $z \in \mathbb{R}$ such that $F^{-1}(z) \cap Y \neq \emptyset$.

2. Let $v^{\infty} \in \mathbb{R}_{+}$and assume that $F^{-1}\left(-c^{\mathrm{T}} A^{-1} v^{\infty}\right) \cap Y \neq \emptyset$. Then, \# $F^{-1}\left(-c^{\mathrm{T}} A^{-1}\right.$ $\left.v^{\infty}\right)=1$ and, for all $x^{0} \in \mathbb{R}^{n}$ and all $v \in L^{\infty}\left(\mathbb{R}_{+}, \mathbb{R}_{+}^{n}\right)$ such that $\lim _{t \rightarrow \infty} v(t)=$ $v^{\infty}$

$$
\lim _{t \rightarrow \infty} x\left(t ; x^{0}, v\right)=-A^{-1}\left(b f\left(y^{\infty}\right)+v^{\infty}\right)=: x^{\infty} \in \mathbb{R}_{+}^{n}
$$

where $\left\{y^{\infty}\right\}=F^{-1}\left(-c^{\mathrm{T}} A^{-1} v^{\infty}\right)$ and $x^{\infty}$ satisfies $c^{\mathrm{T}} x^{\infty}=y^{\infty} \geq 0$.

Proof We extend $f$ to $\mathbb{R}$ by defining

$$
\tilde{f}: \mathbb{R} \rightarrow \mathbb{R}, \quad z \mapsto \begin{cases}f(z), & \text { for } z \geq 0 \\ f(0), & \text { for } z<0\end{cases}
$$

Using (6.2), it is straightforward to show that

$$
\left|\frac{\tilde{f}(z+\xi)-\tilde{f}(\xi)}{z}\right|<\gamma \quad \forall \xi \in Y, \forall z \in \mathbb{R}, z \neq 0 .
$$

Consequently,

$$
|\tilde{f}(z+\xi)-\tilde{f}(\xi)|<\gamma|z| \quad \forall \xi \in Y, \forall z \in \mathbb{R}, z \neq 0
$$

Furthermore, by (6.3),

$$
\gamma|z|-|\tilde{f}(z+\xi)-\tilde{f}(\xi)| \rightarrow \infty \quad \text { as }|z| \rightarrow \infty
$$

Defining $\tilde{F}: \mathbb{R} \rightarrow \mathbb{R}$ by $F(z)=z-\mathbf{G}(0) \tilde{f}(z)$, an application of Proposition 4.1 shows that $\tilde{F}$ is surjective and

$$
\# \tilde{F}^{-1}(z)=1 \text { for every } z \in \mathbb{R} \text { such that } \tilde{F}^{-1}(z) \cap Y \neq \emptyset \text {. }
$$

Now $\tilde{F}(z)<-\mathbf{G}(0) f(0) \leq 0$ for all $z<0$ and so surjectivity of $\tilde{F}$ implies that $\mathbb{R}_{+} \subseteq \tilde{F}\left(\mathbb{R}_{+}\right)=F\left(\mathbb{R}_{+}\right)$. Moreover, let $z \in \mathbb{R}$ be such that $F^{-1}(z) \cap Y \neq \emptyset$. If $w \in$ $F^{-1}(z) \subseteq \mathbb{R}_{+}$, then $z=F(w)=\tilde{F}(w)$, and so $F^{-1}(z) \subseteq \tilde{F}^{-1}(z)$. Consequently, $\tilde{F}^{-1}(z) \cap Y \neq \emptyset$, whence, by $(6.7), \# F^{-1}(z)=\# \tilde{F}^{-1}(z)=1$, completing the proof of statement (1). 
To prove statement (2), let $v^{\infty} \in \mathbb{R}_{+}$be such that $F^{-1}\left(-c^{\mathrm{T}} A^{-1} v^{\infty}\right) \cap Y \neq \emptyset$. It follows from the proof of statement (1) that

$$
F^{-1}\left(-c^{\mathrm{T}} A^{-1} v^{\infty}\right) \cap Y \subseteq \tilde{F}^{-1}\left(-c^{\mathrm{T}} A^{-1} v^{\infty}\right) \cap Y \neq \emptyset
$$

Let $x^{0} \in \mathbb{R}_{+}^{n}$ and let $v \in L^{\infty}\left(\mathbb{R}_{+}, \mathbb{R}_{+}^{n}\right)$ be such that $\lim _{t \rightarrow \infty} v(t)=v^{\infty}$. Setting $x(t):=x\left(t ; x^{0}, v\right)$, it is clear that $x(t) \in \mathbb{R}_{+}^{n}$ for $t \geq 0$, implying that $c^{\mathrm{T}} x(t) \geq 0$ for all $t \geq 0$. Consequently, $x$ is a solution of

$$
\dot{\zeta}=A x+b \tilde{f}\left(c^{\mathrm{T}} \zeta\right)+v
$$

Appealing to (6.5), (6.6) and (6.8), an application of statement (2) of Theorem 4.3 to the Lur'e system (6.9) then shows that

$$
\# \tilde{F}^{-1}\left(-c^{\mathrm{T}} A^{-1} v^{\infty}\right)=1
$$

and

$$
\lim _{t \rightarrow \infty} x(t)=-A^{-1}\left(b \tilde{f}\left(y^{\infty}\right)+v^{\infty}\right)
$$

where $\left\{y^{\infty}\right\}=\tilde{F}^{-1}\left(-c^{\mathrm{T}} A^{-1} v^{\infty}\right)$. By hypothesis, $F^{-1}\left(-c^{\mathrm{T}} A^{-1} v^{\infty}\right) \cap Y \neq \emptyset$ and thus, invoking (6.8) and (6.10), we obtain that

$$
\# F^{-1}\left(-c^{\mathrm{T}} A^{-1} v^{\infty}\right)=1
$$

Finally, since $-c^{\mathrm{T}} A^{-1} v^{\infty} \geq 0$, we have $y^{\infty} \geq 0$, implying that $f\left(y^{\infty}\right)=\tilde{f}\left(y^{\infty}\right)$ and $\left\{y^{\infty}\right\}=F^{-1}\left(-c^{\mathrm{T}} A^{-1} v^{\infty}\right)$. In particular, the right-hand side of (6.11) is equal to $-A^{-1}\left(b f\left(y^{\infty}\right)+v^{\infty}\right)$ and the proof is complete.

As an immediate consequence of Theorem 6.2 we obtain the following result.

Corollary 6.3 Assume that (P1) and (P2) hold and $A$ is Hurwitz. Set $\gamma:=1 / \mathbf{G}(0)$ (where $\gamma:=\infty$ if $\mathbf{G}(0)=0$ ) and assume further that

$$
\left|\frac{f(z)-f(\xi)}{z-\xi}\right|<\gamma \quad \forall(\xi, z) \in \mathbb{R}_{+} \times \mathbb{R}_{+} \text {such that } z \neq \xi,
$$

and (6.3) is satisfied. Then, for every $v^{\infty} \in \mathbb{R}_{+}^{n}, \# F^{-1}\left(-c^{\mathrm{T}} A^{-1} v^{\infty}\right)=1$, with $F$ given by (6.4), and the non-negative Lur'e system (6.1) has the CICS property: for all $x^{0} \in \mathbb{R}_{+}^{n}$ and all $v \in L^{\infty}\left(\mathbb{R}_{+}, \mathbb{R}_{+}^{n}\right)$ with $\lim _{t \rightarrow \infty} v(t)=v^{\infty}$,

$$
\lim _{t \rightarrow \infty} x\left(t ; x^{0}, v\right)=-A^{-1}\left(b f\left(y^{\infty}\right)+v^{\infty}\right)=x^{\infty} \in \mathbb{R}_{+}^{n},
$$

where $\left\{y^{\infty}\right\}=F^{-1}\left(-c^{\mathrm{T}} A^{-1} v^{\infty}\right)$. 
The following lemma (which is an immediate consequence of the mean-value theorem for differentiation) provides a sufficient condition for (6.12) to hold.

Lemma 6.4 Assume that $f: \mathbb{R}_{+} \rightarrow \mathbb{R}_{+}$is continuously differentiable and let $\Delta \subseteq$ $\mathbb{R}_{+}$be a subset which does not have any accumulation points. If

$$
\left|f^{\prime}(z)\right|<\gamma, \quad \forall z \in \mathbb{R}_{+} \backslash \Delta
$$

then (6.12) holds for all $(\xi, z) \in \mathbb{R}_{+} \times \mathbb{R}_{+}$such that $z \neq \xi$.

Example 6.5 Non-negative Lur'e systems of the form (6.1) with

$$
A:=\left(\begin{array}{cccc}
-a_{1} & 0 & \cdots & 0 \\
a_{2} & -a_{3} & \ddots & \vdots \\
& \ddots & \ddots & 0 \\
0 & & a_{2 n-2} & -a_{2 n-1}
\end{array}\right), \quad b:=\left(\begin{array}{c}
b_{1} \\
0 \\
\vdots \\
0
\end{array}\right), \quad c:=\left(\begin{array}{c}
0 \\
\vdots \\
0 \\
1
\end{array}\right),
$$

where $a_{i}>0$ for all $i \in\{1, \ldots, 2 n-1\}$ and $b_{1}>0$, arise in both, population modelling [12] and reaction kinetics, see, for example, [28, Section 7.2]. Obviously, $A$ is Metzler and Hurwitz. In a population dynamics context, the $a_{2 k-1}$ represent mortality rates, the $a_{2 k}$ represent growth rates into the next stage class and $f$ models nonlinear recruitment. The function $v$ could model, for example, immigration effects.

Here we consider the following specific example of the above structure.

$$
A:=\left(\begin{array}{ccc}
-1 & 0 & 0 \\
1 & -1 / 2 & 0 \\
0 & 1 & -2
\end{array}\right), \quad b:=\left(\begin{array}{l}
2 \\
0 \\
0
\end{array}\right), \quad c:=\left(\begin{array}{l}
0 \\
0 \\
1
\end{array}\right) \text {, }
$$

Then,

$$
\mathbf{G}(s)=c^{\mathrm{T}}(s I-A)^{-1} b=\frac{2}{(s+1 / 2)(s+1)(s+2)},
$$

and a routine argument shows that

$$
\|\mathbf{G}\|_{H^{\infty}}=\mathbf{G}(0)=2,
$$

whence $\gamma=1 /\|\mathbf{G}\|_{H^{\infty}}=1 / 2$. We consider the non-negative Lur'e system

$$
\dot{x}=A x+b f\left(c^{\mathrm{T}} x\right)+v
$$

for three different nonlinearities $f: \mathbb{R}_{+} \rightarrow \mathbb{R}_{+}$.

(a) Let $f(z)=z /(z+2)$ for $z \geq 0$. Then, $f^{\prime}(z)=2(z+2)^{-2}$ and so,

$$
f^{\prime}(0)=\frac{1}{2} \text { and } f^{\prime}(z)<\frac{1}{2}, \forall z>0 \text {. }
$$


(a) State components

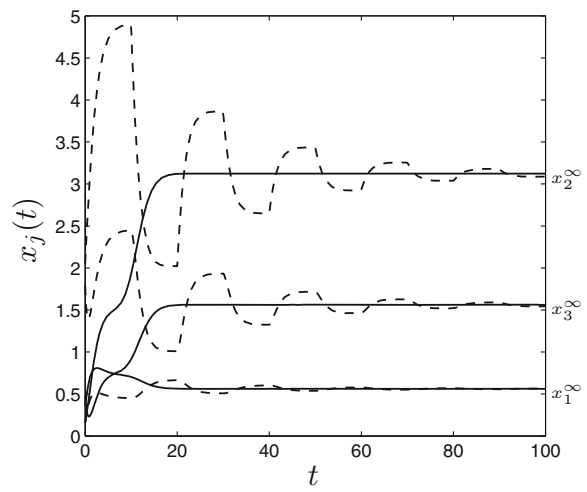

(b) Input signals

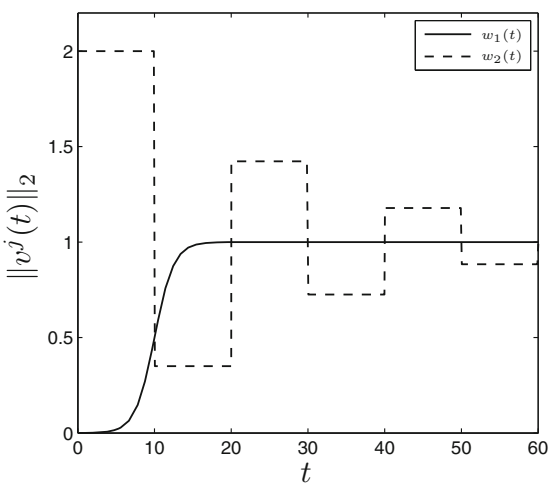

Fig. 4 a State components generated by input signal shown in $\mathbf{b}$ and given by (6.15). The non-zero initial states $x^{0}$ have been chosen randomly

By Lemma 6.4, condition (6.12) holds. Furthermore, (6.3) is trivially satisfied. Consequently, Corollary 6.3 guarantees that (6.14) has the CICS property.

(b) Let $f(z)=1 /(z+2)$ for $z \geq 0$. Then, $f^{\prime}(z)=-(z+2)^{-2}$, and, arguing as in part (a), we see that (6.14) has the CICS property.

Figure 4a displays numerical simulations of the state trajectories generated by the input signals $v^{1}$ and $v^{2}$ given by

$$
v^{j}(t)=\left(\begin{array}{l}
0 \\
1 \\
0
\end{array}\right) w_{j}(t), \quad \text { with } \begin{aligned}
w_{1}(t) & :=\frac{1}{1+\mathrm{e}^{-0.8(t-10)}}, \\
w_{2}(t) & :=1+(-1)^{S(t)}(0.65)^{\lfloor t / 10\rfloor},
\end{aligned}
$$

where $\lfloor z\rfloor \in \mathbb{N}_{0}$ denotes the largest integer less or equal to $z \in \mathbb{R}_{+}$and the "switching function" $S: \mathbb{R}_{+} \rightarrow\{0,1\}$ is defined by

$$
S(t):= \begin{cases}0, & \lfloor t / 10\rfloor \text { even } \\ 1, & \lfloor t / 10\rfloor \text { odd }\end{cases}
$$

The functions $w_{1}$ and $w_{2}$ are plotted in Fig. 4b. Obviously, $w_{1}(t) \rightarrow 1$ and $w_{2}(t) \rightarrow 1$ as $t \rightarrow \infty$, and so

$$
\lim _{t \rightarrow \infty} v^{1}(t)=\lim _{t \rightarrow \infty} v^{2}(t)=(0,1,0)^{\mathrm{T}}=: v^{\infty}
$$

By the CICS property, the limit

$$
\lim _{t \rightarrow \infty} x\left(t ; x^{0}, v^{j}\right)=: x^{\infty}
$$

exists, is independent of $j \in\{1,2\}$ and the initial condition $x^{0}$ and is given by $x^{\infty}=$ $-A^{-1}\left(b f\left(y^{\infty}\right)+v^{\infty}\right)$, where $\left\{y^{\infty}\right\}=F^{-1}\left(-c^{\mathrm{T}} A^{-1} v^{\infty}\right)$ (see Corollary 6.3). The 
condition for $y^{\infty}$ can be expressed in the form

$$
y^{\infty}-\mathbf{G}(0) f\left(y^{\infty}\right)+c^{\mathrm{T}} A^{-1} v^{\infty}=0,
$$

which is a quadratic equation in $y^{\infty}$ and has non-negative solution $y^{\infty}=1.5616$. Now $x^{\infty}$ can be computed and we obtain

$$
x^{\infty}=\left(\begin{array}{l}
0.5615 \\
3.1231 \\
1.5615
\end{array}\right)
$$

see Fig. 4a for an illustration.

(c) Let $f(z)=2 z /(z+1)$ for $z \geq 0$, in which case

$$
f(z)-f(\xi)=\frac{2(z-\xi)}{(z+1)(\xi+1)} \quad \forall(\xi, z) \in \mathbb{R}_{+} \times \mathbb{R}_{+}
$$

Note that, for any $\xi \in[0,3]$, there exists $z \geq 0, z \neq \xi$, such that

$$
\left|\frac{f(z)-f(\xi)}{z-\xi}\right| \geq \frac{1}{2}=\gamma
$$

In particular, for $\xi=3$ :

$$
\frac{f(3)}{3}=\frac{f(0)-f(3)}{0-3}=\frac{1}{2}=\gamma
$$

On the other hand, for every $\xi>3$ :

$$
\left|\frac{f(z)-f(\xi)}{z-\xi}\right|<\frac{1}{2}=\gamma \quad \forall z \geq 0
$$

It is obvious that $z / 2-f(z) \rightarrow \infty$ as $z \rightarrow \infty$, and so, Theorem 6.2 , with $Y:=$ $(3, \infty)$, can be applied to (6.14). To this end, note that the function $F: \mathbb{R}_{+} \rightarrow \mathbb{R}_{+}$ is given by

$$
F(z)=z-\mathbf{G}(0) f(z)=z-\frac{4 z}{z+1},
$$

and so, $F(Y)=(0, \infty)$. Now,

$$
A^{-1}:=\left(\begin{array}{ccc}
-1 & 0 & 0 \\
-2 & -2 & 0 \\
-1 & -1 & -1 / 2
\end{array}\right)
$$


and thus, $-c^{\mathrm{T}} A^{-1}=(1,1,1 / 2)$, showing that

$$
-c^{\mathrm{T}} A^{-1} v^{\infty}>0 \quad \forall v^{\infty} \in \mathbb{R}_{+}^{3} \backslash\{0\} .
$$

Consequently,

$$
F^{-1}\left(-c^{\mathrm{T}} A^{-1} v^{\infty}\right) \cap Y \neq \emptyset \quad \forall v^{\infty} \in \mathbb{R}_{+}^{3} \backslash\{0\} .
$$

Theorem 6.2 guarantees that, for every $v^{\infty} \in \mathbb{R}_{+}^{3} \backslash\{0\}$, there exists $x^{\infty} \in \mathbb{R}_{+}^{3}$ such that $\lim _{t \rightarrow \infty} x\left(t ; x^{0}, v\right)=x^{\infty}$ for all $x^{\infty} \in \mathbb{R}_{+}^{3}$ and all $v \in L^{\infty}\left(\mathbb{R}_{+}, \mathbb{R}_{+}^{3}\right)$ with $\lim _{t \rightarrow \infty} v(t)=v^{\infty}$.

To consider a specific numerical example, let

$$
v^{\infty}=\left(\begin{array}{c}
1 / 4 \\
1 / 4 \\
1
\end{array}\right)
$$

in which case, $-c^{\mathrm{T}} A^{-1} v^{\infty}=1$. Now $F^{-1}(1)=\{2+\sqrt{5}\}$, and so $y^{\infty}=2+$ $\sqrt{5}, f\left(y^{\infty}\right)=(1+\sqrt{5}) / 2$, and

$$
x^{\infty}=-A^{-1}\left(b f\left(y^{\infty}\right)+v^{\infty}\right)=(1+\sqrt{5})\left(\begin{array}{l}
1 \\
2 \\
1
\end{array}\right)+\left(\begin{array}{c}
1 / 4 \\
1 \\
1
\end{array}\right) .
$$

Finally, we comment on input functions $v$ which converge to 0 : there does not exist $x^{\infty}$ such that $\lim _{t \rightarrow \infty} x\left(t ; x^{0}, v\right)=x^{\infty}$ for all $x^{0} \in \mathbb{R}_{+}^{3}$ and all $v \in L^{\infty}\left(\mathbb{R}_{+}, \mathbb{R}_{+}^{3}\right)$ with $\lim _{t \rightarrow \infty} v(t)=0$. Indeed, this follows from the fact that, for $v=0$, the system (6.14) has two equilibria in $\mathbb{R}_{+}^{3}$, namely $(0,0,0)$ and $(3,6,3)^{\mathrm{T}}$. Also note that $F^{-1}(0)=$ $\{0,3\}$ and thus $\# F^{-1}(0)>1$ (cf. Proposition 3.1).

In the context of the Lur'e system discussed in part (c) of Example 6.5, it is interesting to note that the non-zero equilibrium $x^{*}=(3,6,3)^{\mathrm{T}}$ of the uncontrolled system is asymptotically stable with region of attraction equal to $\mathbb{R}_{+}^{3} \backslash\{0\}$. This gives rise to the following question: does $x\left(t ; x^{0}, v\right)$ converge to $x^{*}$ for all non-zero initial conditions $x^{0} \in \mathbb{R}_{+}^{3}$ and all $v \in L^{\infty}\left(\mathbb{R}_{+}, \mathbb{R}_{+}^{3}\right)$ with $\lim _{t \rightarrow \infty} v(t)=0$ ? We shall now state and prove a CICS result which implies that the answer to the question is "yes".

Theorem 6.6 Assume that (P1)-(P3) hold and $A$ is Hurwitz. Set $\gamma:=1 / \mathbf{G}(0)$ and assume further that $f(0)=0$, there exists $y^{*}>0$ such that $f\left(y^{*}\right)=\gamma y^{*},(6.3)$ is satisfied,

$$
\liminf _{z \rightarrow 0} \frac{f(z)}{z}>\gamma
$$

and

$$
\left|\frac{f(z)-f(\xi)}{z-\xi}\right|<\gamma \quad \forall(\xi, z) \in\left[y^{*}, \infty\right) \times(0, \infty), \quad z \neq \xi
$$


Then the following statements hold.

1. The points 0 and $x^{*}:=-\gamma y^{*} A^{-1} b$ are equilibria of the uncontrolled system $\dot{x}=A x+b f\left(c^{\mathrm{T}} x\right)$.

2. The map

$$
F^{*}:\left[y^{*}, \infty\right) \rightarrow \mathbb{R}_{+}, \quad z \mapsto z-\mathbf{G}(0) f(z)
$$

is a bijection.

3. The non-negative Lur'e system (6.1) has the following "quasi-CICS" property: for all $x^{0} \in \mathbb{R}_{+}^{n}$, all $v^{\infty} \in \mathbb{R}_{+}^{n}$ and all $v \in L^{\infty}\left(\mathbb{R}_{+}, \mathbb{R}_{+}^{n}\right)$ such that $\left\|x^{0}\right\|+\|v\|_{L^{\infty}}>0$ and $\lim _{t \rightarrow \infty} v(t)=v^{\infty}$,

$$
\lim _{t \rightarrow \infty} x\left(t ; x^{0}, v\right)=-A^{-1}\left(b f\left(y^{\infty}\right)+v^{\infty}\right)=x^{\infty} \in \mathbb{R}_{+}^{n},
$$

where $y^{\infty}=F^{*-1}\left(-c^{\mathrm{T}} A^{-1} v^{\infty}\right)$. In particular, if $v^{\infty}=0$, then $y^{\infty}=y^{*}$ and $x^{\infty}=x^{*}=-\gamma y^{*} A^{-1} b$.

Proof Since $f(0)=0$, it is obvious that 0 is an equilibrium of $\dot{x}=A x+b f\left(c^{\mathrm{T}} x\right)$. Invoking the hypothesis that $f\left(y^{*}\right)=\gamma y^{*}$, a straightforward calculation shows that $x^{*}$ is also an equilibrium of $\dot{x}=A x+b f\left(c^{\mathrm{T}} x\right)$, completing the proof of statement (1).

To prove statements (2) and (3), let $x^{0}, v^{\infty} \in \mathbb{R}_{+}^{n}$ and $v \in L^{\infty}\left(\mathbb{R}_{+}, \mathbb{R}_{+}^{n}\right)$ be such that $\left\|x^{0}\right\|+\|v\|_{L^{\infty}}>0$ and $\lim _{t \rightarrow \infty} v(t)=v^{\infty}$. We consider two cases: $x^{0} \neq 0$ and $x^{0}=0$.

Case 1: $x^{0} \neq 0$.

Invoking (P1)-(P3) and conditions (6.16) and (6.17), it follows from [6, Proposition 4.12] that there exist $\varepsilon \in\left(0, y^{*}\right)$ and $\tau \geq 0$ such that

$$
c^{\mathrm{T}} x\left(t ; x^{0}, v\right) \geq \varepsilon \quad \forall t \geq \tau .
$$

Consider

$$
\tilde{f}: \mathbb{R} \rightarrow \mathbb{R}, \quad z \mapsto \begin{cases}f\left(z+y^{*}\right)-f\left(y^{*}\right), & \text { for } z \geq-y^{*}+\varepsilon \\ f(\varepsilon)-f\left(y^{*}\right), & \text { for } z<-y^{*}+\varepsilon\end{cases}
$$

and

$$
\tilde{F}: \mathbb{R} \rightarrow \mathbb{R}, \quad z \mapsto z-\mathbf{G}(0) \tilde{f}(z),
$$

and note that, since $f\left(y^{*}\right)=\gamma y^{*}$,

$$
\tilde{F}(z)=z+y^{*}-\mathbf{G}(0) f\left(z+y^{*}\right)=F^{*}\left(z+y^{*}\right) \quad \forall z \geq 0 .
$$

In particular, $\tilde{F}(0)=0$ and, by (6.3) and (6.17),

$$
\tilde{F}(z)>0, \quad \forall z>0 \quad \text { and } \quad \tilde{F}(z) \rightarrow \infty \quad \text { as } z \rightarrow \infty,
$$


implying that $\tilde{F}\left(\mathbb{R}_{+}\right)=\mathbb{R}_{+}$and so

$$
\tilde{F}^{-1}(z) \cap \mathbb{R}_{+} \neq \emptyset \quad \forall z \in \mathbb{R}_{+} .
$$

Next, we prove that

$$
\left|\frac{\tilde{f}(z+\xi)-\tilde{f}(\xi)}{z}\right|<\gamma \quad \forall \xi \in \mathbb{R}_{+}, \forall z \in \mathbb{R}, z \neq 0
$$

To see this, let $\xi \geq 0$. Then, invoking (6.17), we obtain that, for non-zero $z \geq$ $-\left(\xi+y^{*}\right)+\varepsilon$,

$$
\left|\frac{\tilde{f}(z+\xi)-\tilde{f}(\xi)}{z}\right|=\left|\frac{f\left(z+\xi+y^{*}\right)-f\left(\xi+y^{*}\right)}{z+\xi+y^{*}-\left(\xi+y^{*}\right)}\right|<\gamma .
$$

Furthermore, for $z<-\left(\xi+y^{*}\right)+\varepsilon$, we have $|z|=-z>\xi+y^{*}-\varepsilon>0$ and so

$$
\left|\frac{\tilde{f}(z+\xi)-\tilde{f}(\xi)}{z}\right|=\left|\frac{f(\varepsilon)-f\left(\xi+y^{*}\right)}{z}\right|<\left|\frac{f(\varepsilon)-f\left(\xi+y^{*}\right)}{\varepsilon-\left(\xi+y^{*}\right)}\right|<\gamma
$$

where the last inequality follows from (6.17). Therefore, (6.21) holds. Consequently,

$$
|\tilde{f}(z+\xi)-\tilde{f}(\xi)|<\gamma|z| \quad \forall \xi \in \mathbb{R}_{+}, \forall z \in \mathbb{R}, z \neq 0
$$

Moreover, by (6.3),

$$
\gamma|z|-|\tilde{f}(z+\xi)-\tilde{f}(\xi)| \rightarrow \infty \quad \text { as }|z| \rightarrow \infty
$$

Setting $x^{*}:=-A^{-1} b f\left(y^{*}\right)=-\gamma y^{*} A^{-1} b$ and

$$
\tilde{x}(t):=x\left(t+\tau ; x^{0}, v\right)-x^{*} \quad \forall t \geq 0
$$

we have, by (6.18),

$$
c^{\mathrm{T}} \tilde{x}(t)=c^{\mathrm{T}} x\left(t+\tau ; x^{0}, v\right)-y^{*} \geq-y^{*}+\varepsilon \quad \forall t \geq 0,
$$

where we have used that $c^{\mathrm{T}} x^{*}=y^{*}$. Consequently,

$$
\tilde{f}\left(c^{\mathrm{T}} \tilde{x}(t)\right)=f\left(c^{\mathrm{T}} x\left(t+\tau ; x^{0}, v\right)\right)-f\left(y^{*}\right) \quad \forall t \geq 0,
$$

and so, $\tilde{x}$ satisfies

$$
\dot{\tilde{x}}(t)=A \tilde{x}(t)+b \tilde{f}\left(c^{\mathrm{T}} \tilde{x}(t)\right)+\left(\Lambda_{\tau} v\right)(t) \text { for almost all } t \geq 0
$$


where, as before, $\Lambda_{\tau}$ denotes the left-shift by $\tau$. Appealing to (6.20), (6.22) and (6.23), we may apply Theorem 4.3 (with $K=0$ and $Y=\mathbb{R}_{+}$) in the context of the controlled Lur'e system (6.24) and obtain that

$$
\# \tilde{F}^{-1}(z)=1 \quad \forall z \in \mathbb{R}_{+}
$$

and

$$
\lim _{t \rightarrow \infty} \tilde{x}(t)=-A^{-1}\left(b \tilde{f}\left(\tilde{y}^{\infty}\right)+v^{\infty}\right)=: \tilde{x}^{\infty}
$$

where $\left\{\tilde{y}^{\infty}\right\}=\tilde{F}^{-1}\left(-c^{\mathrm{T}} A^{-1} v^{\infty}\right\}$. Equations (6.19), (6.20) and (6.25) show that $F^{*}$ is a bijection. Finally, setting $y^{\infty}:=\tilde{y}^{\infty}+y^{*}$, we obtain from (6.19), that $y^{\infty}=$ $F^{*-1}\left(-c^{\mathrm{T}} A^{-1} v^{\infty}\right)$, and, by $(6.26)$,

$$
\begin{aligned}
x\left(t ; x^{0}, v\right) \rightarrow \tilde{x}^{\infty}+x^{*} & =-A^{-1}\left(b\left(f\left(y^{\infty}\right)-\gamma y^{*}\right)+v^{\infty}\right)-\gamma y^{*} A^{-1} b \\
& =-A^{-1}\left(b f\left(y^{\infty}\right)+v^{\infty}\right)
\end{aligned}
$$

as $t \rightarrow \infty$.

Case 2: $x^{0}=0$.

Then, by hypothesis, $\|v\|_{L^{\infty}}>0$ and thus, there exists $t_{0} \geq 0$ such that

$$
x\left(t_{0} ; 0, v\right)=\int_{0}^{t_{0}} \mathrm{e}^{A\left(t_{0}-s\right)}\left(b f\left(c^{\mathrm{T}} x(s ; 0, v)\right)+v(s)\right) \mathrm{d} s \geq \int_{0}^{t_{0}} \mathrm{e}^{A\left(t_{0}-s\right)} v(s) \mathrm{d} s>0 .
$$

The function $\zeta$ defined by $\zeta(t):=x\left(t+t_{0} ; 0, v\right)$ satisfies

$$
\dot{\zeta}(t)=A \zeta(t)+b f\left(c^{\mathrm{T}} \zeta(t)\right)+\left(\Lambda_{t_{0}} v\right)(t), \quad \zeta(0)=x\left(t_{0} ; 0, v\right)>0 \quad t \geq 0,
$$

and so, by case 1 ,

$$
\lim _{t \rightarrow \infty} x(t ; 0, v)=\lim _{t \rightarrow \infty} \zeta(t)=-A^{-1}\left(b f\left(y^{\infty}\right)+v^{\infty}\right)=x^{\infty},
$$

completing the proof.

Example 6.7 Here we re-visit part (c) of Example 6.5: $A, b$ and $c$ are given by (6.13) and $f(z)=2 z /(z+1)$ for all $z \geq 0$. It is readily verified that $A+b c^{\mathrm{T}}$ is irreducible, that is, condition (P3) is satisfied. We recall that the uncontrolled Lur'e system (6.14) has two equilibria, namely 0 and $x^{*}=(3,6,3)^{\mathrm{T}}$ (the latter being asymptotically stable with domain of attraction $\mathbb{R}_{+}^{3} \backslash\{0\}$, as follows from [6]) and that $\gamma=1 /\|G\|_{H^{\infty}}=1 / 2$. We note that $f(3)=3 / 2=3 \gamma, \liminf _{z \rightarrow 0}(f(z) / z)=f^{\prime}(0)=2$, and

$$
\left|\frac{f(z)-f(\xi)}{z-\xi}\right|=\left|\frac{2}{(z+1)(\xi+1)}\right|<\frac{1}{2} \quad \forall(\xi, z) \in[3, \infty) \times(0, \infty) \text { such that } z \neq \xi .
$$

We may now apply Theorem 6.6 (with $y^{*}=3$ ) and obtain that the Lur'e system under consideration has the quasi-CICS property (in the sense of Theorem 6.6). 
(a) State errors

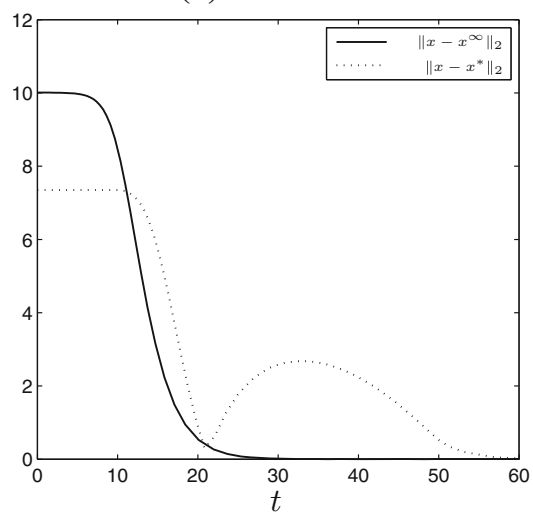

(b) Input signals

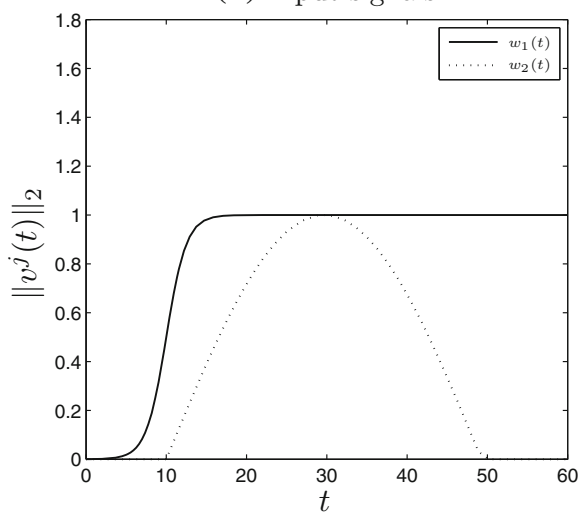

Fig. 5 Numerical simulations: panel a shows the norm of state errors for $x^{0}=0$ corresponding to the input signals shown in panel $\mathbf{b}$

Consider the input signals $v^{1}$ and $v^{2}$ given by $v^{j}(t)=w_{j}(t)(0,1,0)^{\mathrm{T}}$, where

$$
w_{1}(t)=\frac{1}{1+\mathrm{e}^{-0.8(t-10)}} \text { and } w_{2}(t)= \begin{cases}0, & 0 \leq t \leq 10, \\ \sin \left(\frac{2(t-10)}{25}\right), & 10<t \leq 10+\frac{25 \pi}{2} \\ 0, & 10+\frac{25 \pi}{2}<t\end{cases}
$$

see panel (b) of Fig. 5 for an illustration. Note that $v^{1}(t) \rightarrow(0,1,0)^{\mathrm{T}}$ and $v^{2}(t) \rightarrow$ $(0,0,0)^{\mathrm{T}}$ as $t \rightarrow \infty$. By Theorem 6.6, for all $x^{0} \in \mathbb{R}_{+}^{3}, x\left(t ; x^{0}, v^{1}\right) \rightarrow x^{\infty}$ and $x\left(t ; x^{0}, v^{2}\right) \rightarrow x^{*}$ as $t \rightarrow \infty$, where

$$
x^{\infty}=-A^{-1}\left[b f\left(y^{\infty}\right)+\left(\begin{array}{l}
0 \\
1 \\
0
\end{array}\right)\right]=\left(\begin{array}{l}
3.2361 \\
8.4721 \\
4.2361
\end{array}\right) .
$$

Panel (a) of Fig. 5 shows plots of $\left\|x\left(t ; x^{0}, v^{1}\right)-x^{\infty}\right\|_{2}$ (solid line) and $\| x\left(t ; x^{0}, v^{2}\right)-$ $x^{*} \|_{2}$ (dotted line) for $x^{0}=0$. In particular, we see that the state trajectory $x\left(t ; 0, v^{2}\right)$ is at the zero equilibrium for $0 \leq t \leq 10$ since the input $v^{2}$ is zero in this time interval. On the interval $(10,10+25 \pi / 2), v^{2}$ is positive and correspondingly, $x\left(t ; 0, v^{2}\right)$ moves away from the origin and eventually converges to $x^{*}$.

We state a lemma which provides a sufficient condition for (6.17) to hold. The proof of the lemma is given in the Appendix.

Lemma 6.8 Assume that $f: \mathbb{R}_{+} \rightarrow \mathbb{R}_{+}$is continuously differentiable, $f(0)=$ $0, f^{\prime}(z) \geq 0$ for all $z \geq 0, f^{\prime}(0)>\gamma, f^{\prime}$ is non-increasing and $\lim _{z \rightarrow \infty} f^{\prime}(z)<\gamma$. Then there exists $y^{*}>0$ such that $f\left(y^{*}\right)=\gamma y^{*}$ and

$$
\left|\frac{f(z)-f(\xi)}{z-\xi}\right|=\frac{f(z)-f(\xi)}{z-\xi}<\gamma \quad \forall(\xi, z) \in\left[y^{*}, \infty\right) \times(0, \infty), z \neq \xi .
$$


The next lemma specifies an interval such that, for all $\gamma$ in that interval, the so-called Ricker nonlinearity, relevant in the context of models from ecology and population dynamics, satisfies condition (6.17). As with Lemma 6.8, the proof of Lemma 6.9 may be found in the Appendix.

Lemma 6.9 Let $f: \mathbb{R}_{+} \rightarrow \mathbb{R}_{+}$be given by

$$
f(z)=z \mathrm{e}^{-\rho z} \quad \forall z \geq 0
$$

where $\rho>0$ is a parameter, and let $\gamma \in\left[\mathrm{e}^{-2}, 1\right)$. Then $y^{*}:=1 / \rho \ln (1 / \gamma)>0$ is the unique positive solution of $f\left(y^{*}\right)=\gamma y^{*}$ and (6.17) holds.

Example 6.10 Consider the Lur'e system (6.14) in Example 6.5, with $A, b$ and $c$ given by (6.13). Let $f: \mathbb{R}_{+} \rightarrow \mathbb{R}_{+}$be the Ricker nonlinearity given by (6.27), where $\rho>0$ is fixed, but arbitrary. Then conditions (P1)-(P3) hold, $A$ is Hurwitz and $\gamma=1 /\|\mathbf{G}\|_{H^{\infty}}=1 / 2$. Furthermore,

$$
\liminf _{z \rightarrow 0}(f(z) / z)=f^{\prime}(0)=1>1 / 2, \quad z / 2-f(z) \rightarrow \infty \quad \text { as } z \rightarrow \infty
$$

and, by Lemma 6.9, (6.17) is satisfied. Consequently, the Lur'e system (6.14) has the quasi-CICS property in the sense of Theorem 6.6.

\section{Conclusions}

We have considered the CICS property for forced Lur'e systems and have derived a number of necessary and sufficient conditions for CICS. Our motivation has been to study to what extent the appealing CICS property exhibited by linear systems extends to Lur'e systems, and, more broadly, to investigate the effect of convergent additive forcing on the state and output of Lur'e systems. The CICS property for Lur'e systems leads to explicit formulae for the resulting asymptotic state $x^{\infty}$ and output $y^{\infty}$ in terms of the input limit $v^{\infty}$ and enabled us to extend the concept of steady-state gain to Lur'e systems. The theory developed is in the spirit of absolute stability theory and makes use of recent ISS results for Lur'e systems [36]. In particular, several of our sufficient conditions for CICS are reminiscent of the complex Aizerman conjecture, the circle criterion and nonlinear ISS small gain theorems. Finally, for non-negative Lur'e systems, we have derived a "quasi CICS" property which applies to systems which when uncontrolled have two equilibria (one of which is the origin), a common scenario in the context of biological, ecological and chemical models. The proof of our quasi CICS result rests on a recent persistence result [6] for non-negative Lur'e systems.

In future work on forced Lur'e systems, we are planning to investigate the relationship between the concepts of CICS, convergent systems [30] and various notions of incremental stability [2]. One aim is to derive sufficient conditions for the response to (almost) periodic inputs to be asymptotically (almost) periodic. 
Open Access This article is distributed under the terms of the Creative Commons Attribution 4.0 International License (http://creativecommons.org/licenses/by/4.0/), which permits unrestricted use, distribution, and reproduction in any medium, provided you give appropriate credit to the original author(s) and the source, provide a link to the Creative Commons license, and indicate if changes were made.

\section{Appendix A}

In this Appendix, we give proofs of Theorem 2.1, Lemma 6.8 and Lemma 6.9. We also derive the inclusions in (3.1).

Proof of Theorem 2.1. Since $K \in \mathbb{S}_{\mathbb{R}}(A, B, C)$, the matrix $A_{K}=A+B K C$ is Hurwitz. The structured complex stability radius of $A_{K}$ with respect to the weights $B$ and $C$ is defined by

$$
r_{\mathbb{C}}\left(A_{K}, B, C\right):=\inf \left\{\|P\|: P \in \mathbb{C}^{m \times p} \text { such that } A_{K}+B P C \text { is not Hurwitz }\right\}
$$

It is well known $[16,18]$ that

$$
r_{\mathbb{C}}\left(A_{K}, B, C\right)=1 /\left\|\mathbf{G}_{K}\right\|_{H^{\infty}}=\gamma
$$

To prove statements (1) and (2), let $x^{0} \in \mathbb{R}^{n}$ and write $x(t):=x\left(t ; x^{0}, 0\right)$. Obviously, $x$ satisfies $\dot{x}=A_{K} x+B f_{K}(C x)$, where $f_{K}: \mathbb{R}^{p} \rightarrow \mathbb{R}^{m}$ is defined by

$$
f_{K}(z)=f(z)-K z \quad \forall z \in \mathbb{R}^{p} .
$$

By hypothesis, $\left\|f_{K}(z)\right\|<\gamma\|z\|$ for all non-zero $z \in \mathbb{R}^{p}$ and thus the claim follows from (A.1) and [18, Theorem 5.6.22] or [17, Corollary 3.15]. Moreover, by (A.1), $\mathbb{B}_{\mathbb{C}}(K, \gamma) \subseteq \mathbb{S}_{\mathbb{C}}(A, B . C)$, and thus, statement (2) is a consequence of [36, Theorem 3.2].

We proceed to prove statement (3). To this end, let $f: \mathbb{R}^{p} \rightarrow \mathbb{R}^{m}$ be locally Lipschitz and such that $f(0)=0$. We show that the equilibrium pair $(0,0)$ of (1.1) is ISS. Let $x^{0} \in \mathbb{R}^{n}$ and $v \in L_{\text {loc }}^{\infty}\left(\mathbb{R}_{+}, \mathbb{R}^{n}\right)$ be arbitrary and set $x(t):=x\left(t ; x^{0}, v\right)$. Then $\dot{x}=A_{K} x+B f_{K}(C x)+v$, where $f_{K}$ is defined by (A.2). Thus, by the variation-ofparameters formula,

$$
x(t)=\mathrm{e}^{A_{K} t} x^{0}+\int_{0}^{t} \mathrm{e}^{A_{K}(t-s)}\left(B f_{K}(C x(s))+v(s)\right) \mathrm{d} s \quad \forall t \in[0, \omega),
$$

where $0<\omega \leq \infty$ and $[0, \omega)$ is the maximal interval of existence of the forward solution $x$. Note that, since $f$ is not necessarily affine linearly bounded, finite escape time cannot be ruled out at this stage. Now $C \mathrm{e}^{A_{K} t} B$ is the inverse Laplace transform of $\mathbf{G}_{K}$ and hence $C \mathrm{e}^{A_{K} t} B=0$ for all $t \in \mathbb{R}$. Consequently, it follows from (A.3),

$$
C x(t)=C \mathrm{e}^{A_{K} t} x^{0}+\int_{0}^{t} C \mathrm{e}^{A_{K}(t-s)} v(s) \mathrm{d} s \quad \forall t \in[0, \omega) .
$$


Since $A_{K}$ is Hurwitz, it follows that $C x$ is bounded on any bounded subinterval of $[0, \omega)$ and thus, by (A.3), $x$ is also bounded on any bounded subinterval of $[0, \omega)$. We may, therefore, conclude that $\omega=\infty$.

By the Hurwitz property of $A_{K}$, there exist $M \geq 1$ and $\mu>0$ such that

$$
\left\|\mathrm{e}^{A_{K} t}\right\| \leq M \mathrm{e}^{-\mu t} \quad \forall t \geq 0
$$

Combining this with (A.4) shows that there exist positive constants $M_{1}$ and $M_{2}$ such that, for all $x^{0} \in \mathbb{R}^{n}$ and $v \in L_{\mathrm{loc}}^{\infty}\left(\mathbb{R}_{+}, \mathbb{R}^{n}\right)$,

$$
\|C x(t)\| \leq M_{1} \mathrm{e}^{-\mu t}\left\|x^{0}\right\|+M_{2}\|v\|_{L^{\infty}(0, t)} \quad \forall t \geq 0
$$

Moreover, let $\eta \in \mathcal{K}$ be such that

$$
\|B\|\left\|f_{K}(z)\right\| \leq \eta(\|z\|) \quad \forall z \in \mathbb{R}^{p}
$$

The existence of such a function $\eta$ follows from the continuity of $f_{K}$ and the fact that $f_{K}(0)=0$. Invoking (A.5) and (A.6), we obtain

$$
\|B\|\left\|f_{K}(C x(t))\right\| \leq \eta_{1}\left(\mathrm{e}^{-\mu t}\left\|x^{0}\right\|\right)+\eta_{2}\left(\|v\|_{L^{\infty}(0, t)}\right) \quad \forall t \geq 0
$$

where the $\mathcal{K}$-functions $\eta_{1}$ and $\eta_{2}$ are defined by $\eta_{1}(s)=\eta\left(2 M_{1} s\right)$ and $\eta_{2}(s)=$ $\eta\left(2 M_{2} s\right)$.

Next we estimate the term $I(t):=\int_{0}^{t} \mathrm{e}^{A_{K}(t-s)} B f_{K}(C x(s)) \mathrm{d} s$. To this end, writing

$$
I(t)=\int_{0}^{t / 2} \mathrm{e}^{A_{K}(t-s)} B f_{K}(C x(s)) \mathrm{d} s+\int_{t / 2}^{t} \mathrm{e}^{A_{K}(t-s)} B f_{K}(C x(s)) \mathrm{d} s,
$$

we note that, by (A.7),

$$
\begin{aligned}
\|I(t)\| \leq & M \mathrm{e}^{-(\mu / 2) t}\left(\eta_{1}\left(\left\|x^{0}\right\|\right)+\eta_{2}\left(\|v\|_{L^{\infty}(0, t)}\right)\right) \int_{0}^{t / 2} \mathrm{e}^{-\mu(t / 2-s)} \mathrm{d} s \\
& +\frac{M}{\mu}\left(\eta_{1}\left(\mathrm{e}^{-(\mu / 2) t}\left\|x^{0}\right\|\right)+\eta_{2}\left(\|v\|_{L^{\infty}(0, t)}\right)\right) \quad \forall t \geq 0 .
\end{aligned}
$$

Consequently,

$$
\|I(t)\| \leq \frac{M}{\mu}\left(\mathrm{e}^{-(\mu / 2) t} \eta_{1}\left(\left\|x^{0}\right\|\right)+\eta_{1}\left(\mathrm{e}^{-(\mu / 2) t}\left\|x^{0}\right\|\right)+\frac{2 M}{\mu} \eta_{2}\left(\|v\|_{L^{\infty}(0, t)}\right) \quad \forall t \geq 0 .\right.
$$

Furthermore,

$$
\left\|\int_{0}^{t} \mathrm{e}^{A_{K}(t-s)} v(s) \mathrm{d} s\right\| \leq \frac{M}{\mu}\|v\|_{L^{\infty}(0, t)} \quad \forall t \geq 0,
$$


and therefore, by (A.3),

$$
\begin{aligned}
\|x(t)\| \leq & M \mathrm{e}^{-\mu t}\left\|x^{0}\right\|+\frac{M}{\mu}\left(\mathrm{e}^{-(\mu / 2) t} \eta_{1}\left(\left\|x^{0}\right\|\right)+\eta_{1}\left(\mathrm{e}^{-(\mu / 2) t}\left\|x^{0}\right\|\right)\right) \\
& +\frac{M}{\mu}\left(2 \eta_{2}\left(\|v\|_{L^{\infty}(0, t)}\right)+\|v\|_{L^{\infty}(0, t)}\right) \quad \forall t \geq 0 .
\end{aligned}
$$

Hence, defining $\psi \in \mathcal{K} \mathcal{L}$ and $\varphi \in \mathcal{K}$ by

$$
\begin{aligned}
\psi(s, t) & :=M \mathrm{e}^{-\mu t} s+\frac{M}{\mu}\left(\mathrm{e}^{-(\mu / 2) t} \eta_{1}(s)+\eta_{1}\left(\mathrm{e}^{-(\mu / 2) t} s\right)\right) \text { and } \\
\varphi(s) & :=\frac{M}{\mu}\left(2 \eta_{2}(s)+s\right)
\end{aligned}
$$

respectively, we conclude that, for every $x^{0} \in \mathbb{R}^{n}$ and every $v \in L_{\text {loc }}^{\infty}\left(\mathbb{R}_{+}, \mathbb{R}^{n}\right)$,

$$
\left\|x\left(t ; x^{0}, v\right)\right\|=\|x(t)\| \leq \psi\left(\left\|x^{0}\right\|, t\right)+\varphi\left(\|v\|_{L^{\infty}(0, t)}\right) \quad \forall t \geq 0,
$$

showing that the equilibrium pair $(0,0)$ of $(1.1)$ is ISS.

Proof of (3.1) To prove the first inclusion, let $z \in \operatorname{im} C$, so that $z=C w$ for some $w \in \mathbb{R}^{n}$. Then

$$
\begin{aligned}
F_{K}(z) & =F_{K}(C w)=C w-\mathbf{G}_{K}(0)(f(C w)-K C w) \\
& =C\left[w+A_{K}^{-1} B(f(C w)-K C w)\right] \in \operatorname{im} C .
\end{aligned}
$$

For the proof of the second inclusion assume that $z \in F_{K}^{-1}(\operatorname{im} C)$, so that $F_{K}(z)=C w$, for some $w \in \mathbb{R}^{n}$. Then

$$
z=F_{K}(z)+\mathbf{G}_{K}(0)(f(z)-K z)=C\left[w-A_{K}^{-1} B(f(z)-K z)\right] \in \operatorname{im} C,
$$

as required.

Proof of Lemma 6.8. It follows immediately from the hypotheses that there exist $y^{*}>$ $y_{*}>0$ such that $f\left(y^{*}\right)=\gamma y^{*}, f^{\prime}\left(y_{*}\right)=\gamma, f^{\prime}(z)>\gamma$ if $z \in\left[0, y_{*}\right)$ and $f^{\prime}(z)<\gamma$ if $z>y_{*}$. We consider two cases.

Case 1: $\xi \geq y^{*}$ and $z>y_{*}, z \neq \xi$.

In this case,

$$
|f(z)-f(\xi)|=\left|\int_{\xi}^{z} f^{\prime}(s) \mathrm{d} s\right|<\gamma|z-\xi| .
$$

Case 2: $\xi \geq y^{*}$ and $z \in\left(0, y_{*}\right]$.

Note that, by Case $1,\left|f\left(y^{*}\right)-f(\xi)\right| \leq \gamma\left|y^{*}-\xi\right|$ and thus,

$$
|f(z)-f(\xi)| \leq\left|f(z)-f\left(y^{*}\right)\right|+\gamma\left|y^{*}-\xi\right|=\gamma\left|y^{*}-\xi\right|+\gamma y^{*}-f(z) .
$$


Now

$$
f(z)=\int_{0}^{z} f^{\prime}(s) \mathrm{d} s>\gamma z
$$

and we conclude that

$$
|f(z)-f(\xi)|<\gamma\left|\xi-y^{*}\right|+\gamma\left(y^{*}-z\right)=\gamma|z-\xi| .
$$

In both cases we have

$$
|f(z)-f(\xi)|<\gamma|z-\xi|,
$$

completing the proof.

Proof of Lemma 6.9. Note that, as $z$ decreases from $2 / \rho$ to $0, f(z) / z=\mathrm{e}^{-\rho z}$ increases strictly monotonically from $\mathrm{e}^{-2}$ to 1 . Consequently, for every, $\gamma \in\left[\mathrm{e}^{-2}, 1\right)$, there exists a unique $y^{*} \in(0,2 / \rho]$ such that $f\left(y^{*}\right)=\gamma y^{*}$.

To prove that (6.17) holds, we initially assume that $\gamma \in\left(\mathrm{e}^{-2}, 1\right)$ and deal with the case $\gamma=\mathrm{e}^{-2}$ separately. We proceed in several steps.

Step 1 A routine calculation shows

$$
\min _{z \geq 0} f^{\prime}(z)=f^{\prime}(2 / \rho)=-\mathrm{e}^{-2}, \quad f^{\prime}(z)>-\mathrm{e}^{-2} \forall z \geq 0, z \neq 2 / \rho .
$$

Since $\gamma>\mathrm{e}^{-2}$, we conclude that

$$
f^{\prime}(z)>-\gamma \quad \forall z \geq 0
$$

and hence, by the mean-value theorem for differentiation,

$$
\frac{f(z)-f(\xi)}{z-\xi}>-\gamma \quad \forall z \geq 0, \forall \xi \geq y^{*}, z \neq \xi .
$$

Step 2 Note that $f^{\prime}(z)<\gamma$ for all $z \in\left[y^{*}, 2 / \rho\right]$ (which follows because $f^{\prime}(0)>\gamma, f^{\prime}$ is non-increasing on $[0,2 / \rho]$ and $\left.f\left(y^{*}\right)=\gamma y^{*}\right)$. Since $f^{\prime}(z)<0<\gamma$ for all $z>2 / \rho$, we obtain, via the mean-value theorem for differentiation,

$$
\frac{f(z)-f(\xi)}{z-\xi}<\gamma \quad \forall z, \xi \geq y^{*}, z \neq \xi .
$$

Step 3 Let $\xi \geq y^{*}$ and $z \in\left(0, y^{*}\right)$. By (A.9) and (A.10),

$$
\left|f\left(y^{*}\right)-f(\xi)\right| \leq \gamma\left|y^{*}-\xi\right|,
$$


Moreover, invoking again (A.9) and using that $f(z)>\gamma z$, we obtain

$$
-\gamma<\frac{f\left(y^{*}\right)-f(z)}{y^{*}-z}<\frac{\gamma\left(y^{*}-z\right)}{y^{*}-z}=\gamma
$$

and so

$$
\left|f(z)-f\left(y^{*}\right)\right|<\gamma\left|z-y^{*}\right| .
$$

Now $|f(z)-f(\xi)| \leq\left|f(z)-f\left(y^{*}\right)\right|+\left|f\left(y^{*}\right)-f(\xi)\right|$, and thus, by (A.11) and (A.12),

$$
|f(z)-f(\xi)|<\gamma\left|z-y^{*}\right|+\gamma\left|y^{*}-\xi\right|=\gamma\left(\left|z-y^{*}\right|+\left|y^{*}-\xi\right|\right) .
$$

Since $z<y^{*} \leq \xi$, we have $\left|z-y^{*}\right|+\left|y^{*}-\xi\right|=|z-\xi|$ and conclude

$$
\left|\frac{f(z)-f(\xi)}{z-\xi}\right|<\gamma \quad \forall z \in\left(0, y^{*}\right), \forall \xi \geq y^{*}
$$

Step 4 Combining (A.9), (A.10) and (A.13), we arrive at

$$
\left|\frac{f(z)-f(\xi)}{z-\xi}\right|<\gamma \quad \forall(\xi, z) \in\left[y^{*}, \infty\right) \times(0, \infty) \text { such that } z \neq \xi,
$$

which is (6.17).

Finally, let us consider the case wherein $\gamma=\mathrm{e}^{-2}$. Then $y^{*}=2 / \rho$ and so $f^{\prime}\left(y^{*}\right)=$ $f^{\prime}(2 / \rho)=-\mathrm{e}^{-2}=-\gamma$, implying that (A.8) does not hold for $z=y^{*}$. We will show that nevertheless (A.9) remains valid in this case. To this end, note that

$$
f^{\prime}(z)>-\gamma \quad \forall z \geq 0, z \neq y^{*}
$$

From the mean-value theorem we immediately obtain that

$$
\frac{f(z)-f(\xi)}{z-\xi}>-\gamma \quad \forall z, \xi \geq y^{*}, z \neq \xi
$$

Let $z \in\left(0, y^{*}\right)$ and $\xi \geq y^{*}$. Then

$$
f(z)-f(\xi)=f(z)-f\left(y^{*}\right)+f\left(y^{*}\right)-f(\xi) \leq f(z)-f\left(y^{*}\right)+\gamma\left(\xi-y^{*}\right),
$$

where we have used (A.15). Yet another application of the mean-value theorem shows that there exists $\theta \in\left(z, y^{*}\right)$ such that

$$
\frac{f(z)-f\left(y^{*}\right)}{z-y^{*}}=f^{\prime}(\theta)>-\gamma
$$


where the inequality follows from (A.14). Consequently, $f(z)-f\left(y^{*}\right)<\gamma\left(y^{*}-z\right)$, and so, invoking (A.16), we arrive at $f(z)-f(\xi)<\gamma(\xi-z)$, or equivalently,

$$
\frac{f(z)-f(\xi)}{z-\xi}>-\gamma .
$$

We have now shown that (A.9) still holds in the case wherein $\gamma=\mathrm{e}^{-2}$. Steps 2-4 remain valid without change and, therefore, we conclude that (6.17) is satisfied in the case under consideration, completing the proof.

\section{References}

1. Aminzarey Z, Sontag ED (2014) Contraction methods for nonlinear systems: a brief introduction and some open problems. In: Proceedings of 53rd IEEE conference on decision and control (CDC), Los Angeles, California, pp 3835-3847

2. Angeli D (2002) A Lyapunov approach to incremental stability properties. IEEE Trans Autom Control 47:410-421

3. Angeli D, De Leenheer P, Sontag ED (2004) A small-gain theorem for almost global convergence of monotone systems. Syst Control Lett 52:407-414

4. Arcak M, Teel A (2002) Input-to-state stability for a class of Lurie systems. Automatica 38:1945-1949

5. Berman A, Plemmons RJ (1994) Nonnegative Matrices in the Mathematical Sciences. Classics in Applied Mathematics. SIAM, Philadelphia

6. Bill A, Guiver C, Logemann H, Townley S (2016) Stability of non-negative Lur'e systems. SIAM J Control Optim 54:1176-1211

7. Chaillet A, Pogromsky AY, Rüffer BS (2013) A Razumikhin approach for the incremental stability of delayed nonlinear systems. In: Proceedings of 52nd IEEE conference on decision and control (CDC), Florence, Italy, pp 1596-1601

8. Courchamp F, Berec L, Gascoigne J (2008) Allee effects in ecology and conservation. Environ Conserv 36:80-85

9. Dashkovskiy SN, Efimov DV, Sontag ED (2011) Input-to-state stability and allied system properties. Autom Remote Control 72:1579-1614

10. Dashkovskiy SN, Rüffer BS, Wirth FR (2007) An ISS small gain theorem for general networks. Math Control Signals Syst 19:93-122

11. Desoer CA, Vidyasagar M (1975) Feedback systems: input-output properties. Academic Press, New York

12. Gouzé JL, Rapaport A, Hadj-Sadok HZ (2000) Interval observers for uncertain biological systems. Ecol Model 133:45-56

13. Haddad WM, Bernstein DS (1993) Explicit construction of quadratic Lyapunov functions for the small gain, positivity, circle, and Popov theorems and their application to robust stability (part I: continuoustime theory). Int J Robust Nonlinear Control 3:313-339

14. Haddad WM, Chellaboina V (2008) Nonlinear dynamical systems and control. Princeton University Press, Princeton

15. Haddad WM, Chellaboina V, Hui Q (2010) Non-negative and compartmental dynamical systems. Princeton University Press, Princeton

16. Hinrichsen D, Pritchard AJ (1986) Stability radius for structured perturbations and the algebraic Riccati equation. Syst Control Lett 8:105-113

17. Hinrichsen D, Pritchard AJ (1992) Destabilization by output feedback. Differ Integral Equ 5:357-386

18. Hinrichsen D, Pritchard AJ (2005) Mathematical systems theory I. Springer, Berlin

19. Jayawardhana B, Logemann H, Ryan EP (2009) Input-to-state stability of differential inclusions with applications to hysteretic and quantized feedback systems. SIAM J Control Optim 48:1031-1054

20. Jayawardhana B, Logemann H, Ryan EP (2011) The circle criterion and input-to-state stability: new perspectives on a classical result. IEEE Control Syst Mag 31:32-67 
21. Jiang ZP, Teel AR, Praly L (1994) Small-gain theorem for ISS systems and applications. Math Control Signals Syst 7:95-120

22. Kellet CM (2014) A compendium of comparison function results. Math Control Signals Syst 26:339374

23. Khalil HK (2002) Nonlinear systems, 3rd edn. Prentice-Hall, Upper Saddle River

24. Liberzon MR (2006) Essays on absolute stability theory. Autom Remote Control 67:1610-1644

25. Logemann H, Ryan EP (2003) Non-autonomous systems: asymptotic behaviour and weak invariance principles. J Differ Equ 189:440-460

26. Luenberger DG (1979) Introduction to dynamical systems. Wiley, New York

27. Mayer CD (2000) Matrix analysis and applied linear algebra. SIAM, Philadelphia

28. Murray JD (2002) Mathematical biology I, 3rd edn. Springer, New York

29. Partington JP (2004) Linear operators and linear systems. Cambridge University Press, Cambridge

30. Pavlov A, van de Wouw N, Nijmeijer H (2006) Uniform output regulation of nonlinear systems: a convergent dynamics approach. Birkhäuser Boston, Boston

31. Pogromsky AY, Matveev MS (2013) A non-quadratic criterion for stability of forced oscillations. Syst Control Lett 62:408-412

32. Renardy M, Rogers RC (1993) An introduction to partial differential equations. Springer, New York

33. Rüffer BS, van de Wouw N, Mueller M (2013) Convergent systems vs. incremental stability. Syst Control Lett 62:277-285

34. Ryan EP, Sontag ED (2006) Well-defined steady-state response does not imply CICS. Syst Control Lett 55:707-710

35. Sandberg IW, Kramer Johnson K (1992) Steady-state errors in nonlinear control systems. IEEE Trans Autom Control 37:1985-1989

36. Sarkans E, Logemann H (2015) Input-to-state stability for Lur'e systems. Math Control Signals Syst 27:439-465

37. Smith HL, Thieme HR (2013) Persistence and global stability for a class of discrete time structured population models. Discrete Contin Dyn Syst 33:4627-4646

38. Sontag ED (1989) Smooth stabilization implies coprime factorization. IEEE Trans Autom Control $34: 435-443$

39. Sontag ED (1998) Comments on integral variants of ISS. Syst Control Lett 34:93-100

40. Sontag ED (2002) Asymptotic amplitudes and Cauchy gains: a small-gain principle and an application to inhibitory biological feedback. Syst Control Lett 47:167-179

41. Sontag ED (2003) A remark on the converging-input converging-state property. IEEE Trans Autom Control 48:313-314

42. Sontag ED (2006) Input to state stability: basic concepts and results. In: Nistri P, Stefani G (eds) Nonlinear and optimal control theory. Springer, Berlin, pp 163-220

43. Sontag ED, Ingalls B (2002) A small-gain theorem with applications to input/output systems, incremental stability, detectability, and interconnections. J Frankl Inst 339:211-229

44. Townley S, Rebarber R, Tenhumberg B (2012) Feedback control systems analysis of density dependent population dynamics. Syst Control Lett 61:309-315

45. Vidyasagar M (1993) Nonlinear systems analysis, 2nd edn. Prentice-Hall, Englewood Cliffs

46. Yakubovich VA (1964) Matrix inequalities method in stability theory for nonlinear control systems: I. Absolute stability of forced vibrations. Autom Remote Control 7:905-917

47. Yakubovich VA, Leonov GA, Gelig AKh (2004) Stability of stationary sets in control systems with discontinuous nonlinearities. World Scientific, Singapore 